\title{
Instantons of Two-Dimensional Fermionic Effective Actions by Inverse Scattering Transformation
}

\author{
J. Avan and H. J. de Vega \\ Laboratoire de Physique Théorique et Hautes Energies`, Université Pierre et Marie Curie, \\ F-75230 Paris Cedex 05, France
}

\begin{abstract}
Effective actions, containing the logarithm of a functional Dirac determinant, appear in $1 / N$ expansions of fermionic theories ( $N$ being the number of flavours). We introduce a method to find symmetric solutions of the corresponding non-linear and non-local saddle-point equations. This method consists in using the scattering data of the rotationally symmetric Dirac equation in two dimensions with the angular mometum as a spectral parameter. We apply the method to fermionic theories with scalar and pseudoscalar quartic couplings. The effective action that generates the $1 / N$ expansion admits a closed form in terms of the scattering data only in the particular case when the model is integrable (Gross-Neveu and Chiral GrossNeveu model). No instanton solutions are present in these two particular cases. This fact, together with the exact results for the $S$-matrix and form factors, suggests that the $1 / N$ expansion could be convergent. In the general case, the quantum model has an additional dimensionless parameter $g_{R} \cdot g_{R} \rightarrow \pm \infty$ gives the Chiral Gross-Neveu model. When $g_{R}>0$, tachyons appear. For $g_{R} \rightarrow 0^{-}$, and $g_{R} \rightarrow-\infty$, generically complex-action instantons exists, indicating a possibly Borel-summable $1 / N$ expansion.
\end{abstract}

\section{Introduction}

Effective actions play an important role in many problems of quantum field theory [1]. At the one-loop level they have the general structure:

$$
\log \operatorname{det} \mathcal{O}(v, w, \ldots)+\int d^{v} x L(v, w, \partial v, \partial w, \ldots),
$$

where $\mathcal{O}$ is a partial differential operator and $L$ a polynomial. Both $\mathcal{O}$ and $L$ contain the fields $v, w, \ldots$ and their first derivatives. So the effective action contains a nonlocal functional of the fields [the Logdet in (1.1)] plus a local functional (the

* Laboratoire associé au CNRS LA280 
polynomial part $L$ ). The saddle-point equations of such effective actions lead to non-local and non-polynomial equations.

In a series of papers we were able to study effective actions in bosonic field theories. This was the case for the $N$-dimensional anharmonic oscillator $\left(\mathbf{x}^{2}\right)^{2}$ in the framework of the $1 / N$ perturbation series $[2,3]$, generalized to an $O(N)$ invariant potential $V\left(\mathbf{x}^{2}\right)$ [4], and to $\left(\boldsymbol{\Phi}^{2}\right)^{2}$ field theory in 2,3 , and 4 euclidean space dimensions [5]. The technique used was the inverse scattering transformation. The fields $v, w, \ldots$ were replaced by the scattering variables associated to them through the auxiliary linear problem

$$
\mathcal{O}(v, w, \ldots) \varphi=0 .
$$

This can always be done provided the fields depend only on one variable, that can be the distance to the origin (spherically symmetric fields) [5-7] or one of the cartesian coordinates in euclidean space [8]. In terms of the scattering data the In $\operatorname{det}(\mathcal{O})$ becomes a local functional. In some cases the integral-polynomial part of $S_{\text {eff }}: \int d^{v} x L(v)$ is also a local functional of the scattering data, indicating that the saddle point equations associated to $S_{\text {eff }}$ are completely integrable. This happened for the $N$-dimensional anharmonic oscillator $[2,3]$, the non-linear sigma model in 2 dimensions $[5,9]$, and the massless $\left(\Phi^{2}\right)^{2}$ theory in 4 dimensions [5]. Here the use of the scattering data allowed us to find saddle points of the effective action in every case (integrable or not). In the integrable cases they were obtained in closed form. These instantons rule the large orders of perturbation in $1 / N$ for $\left(\Phi^{2}\right)^{2}[2-6,10]$. In the case of the $g\left(\Phi^{2}\right)^{2}$ theory in 4 dimensions, the instanton found for $g_{\text {Ren }}\langle 0[5]$ connected the $\langle\Phi\rangle=0$ vacuum with an infinite field configuration, showing the unstable character of the theory and providing an estimate for its lifetime.

This paper deals with fermionic effective actions; namely, when $\mathcal{O}$ is a Diractype differential operator. This case has a considerable interest since one finds fermionic effective actions of the type (1.1) after integration over anti-commuting variables in theories like Yukawa, QED and QCD. Analogous actions appear in two-dimensional fermionic self-coupled models like:

$$
\mathscr{L}=\bar{\Psi} \not \partial \Psi+g_{1}(\bar{\Psi} \Psi)^{2}+g_{2}\left(\bar{\Psi}_{\gamma_{5}} \Psi\right)^{2},
$$

where $\Psi=\left(\Psi_{1} \ldots \Psi_{N}\right)$ is an $N$-component Dirac field. One constructs here (Sect. II) a $1 / N$ expansion through the introduction of auxiliary fields $v$ and $w$. This leads after renormalization to an effective action:

$$
\begin{aligned}
S_{\text {eff }}= & \log \operatorname{det}\left(\frac{-\not \partial+m+v+i \gamma_{5} w}{-\not \partial+m}\right) \\
& +\frac{\Gamma(1-v / 2)}{(2 \pi)^{v / 2}} 2^{v / 2-1} \int_{-\infty}^{+\infty}\left(v^{2}+2 m v+w^{2}\right) d^{v} x+\frac{1}{g_{R}} \int_{-\infty}^{+\infty} w^{2} d^{v} x,
\end{aligned}
$$

where $1 / g_{R}$ must be kept negative so as to get a tachyon free propagator for the field $w$. The renormalized mass $m$ is generated dynamically [the classical Lagrangian is scale-invariant; see formula (1.3)]. $g_{R}$ is a renormalized coupling constant. Here a 
fermion-antifermion bound state appears in the $w$-channel. It becomes massless (infraparticle) $[11,14]$ in the chiral symmetry limit $1 / g_{R} \rightarrow 0$. The saddle point equation for the effective action reads:

$$
\begin{gathered}
\operatorname{Tr}\left\langle x\left|\frac{1}{-\not \partial+m+v+i \gamma_{5} w}\right| x\right\rangle+\frac{\Gamma(1-v / 2)}{(4 \pi)^{v / 2}} 2^{v / 2}(v+m)=0, \\
\operatorname{Tr}\left\langle x\left|\frac{1}{-\not \partial+m+v+i \gamma_{5} w} \cdot i \gamma_{5}\right| x\right\rangle+\frac{\Gamma(1-v / 2)}{(4 \pi)^{v / 2}} 2^{v / 2} w=0 .
\end{gathered}
$$

Here $v$ and $w$ are a priori complex potentials, and $\operatorname{Tr}$ stands for trace on chirality indices. We shall look for rotationally invariant stationary points. They usually dominate in the semi-classical regime (large $N$ in the present case) [12]. For such fields the inverse scattering transformation in the angular momentum for the Dirac equation is the appropriate tool, as was the case (Schrödinger equation) for the bosonic fields [5]. When $v$ and $w$ depend only on the radial coordinate $r$, one can expand the ln det in Eq. (1.1) in partial waves as

$$
\ln \operatorname{det} \mathscr{D}=\sum_{J=-\infty}^{+\infty} \ln \operatorname{det} D_{I I}(J)
$$

where $D_{I I}(J)$ stands for the radial Dirac operator in two dimensions:

$$
D_{I I}(J)=\left(\begin{array}{cc}
m+v+i w & -\partial_{r}-\frac{J}{r} \\
-\partial_{r}+\frac{J}{r} & m+v-i w
\end{array}\right) .
$$

The spectrum of $D_{I I}(J)$ can be analysed (Sect. III), with the help of the Jost function, defined as:

$$
F(J)=\lim _{r \rightarrow+\infty} e^{-m r} \varphi^{(1)}(r, J)
$$

where $\varphi^{(1)}$ is the upper component of the regular solution (near $r=0$ ) of $D_{I I} \varphi=0$. This spectrum consists of a continuum part for purely imaginary $J$, plus a discrete part $\left\{J_{K}\right\}$. This discrete spectrum corresponds to solutions regular at $r=0$ and $r=+\infty$, or equivalently to zeroes of the Jost function. It can be shown that the following set of scattering data (S.D.) is in one-to-one correspondence with the fields $v(r)$ and $w(r)$ :

$$
\text { S.D. }=\left\{D(\tau), \tau \in \mathbb{R} ; J_{K}^{ \pm}, c_{K}, K=1 \ldots N_{B}^{ \pm}\right\} \quad\left(\operatorname{Re} J_{K}^{+}>0 ; \operatorname{Re} J_{K}^{-}<0\right),
$$

where

$$
D(\tau)=\frac{F(i \tau+0) F(i \tau-0)}{F_{0}(i \tau+0) F_{0}(i \tau-0)}
$$

$F_{0}$ is the Jost function for $v=w=0,\left\{J_{K}\right\}$ are the zeroes of the Jost function, and $\left\{c_{K}\right\}$ are the normalization coefficients of the corresponding wave function. It can then be shown that: 


$$
\begin{aligned}
\frac{F(J)}{F_{0}(J)}= & \prod_{\operatorname{Re} J_{K}>0} \frac{J-J_{K}^{+}}{J+J_{K}^{+}} \exp -\frac{1}{2 \pi} \int_{-\infty}^{+\infty} \frac{d \tau}{(i \tau-J)} \\
& \cdot\left(\ln D(\tau)+\sum_{K} \ln \left(\frac{i \tau+J_{K}}{i \tau-J_{K}}\right)\right)(\operatorname{Re} J>0), \\
\frac{F(J)}{F_{0}(J)}= & \prod_{\operatorname{Re} J_{K}<0} \frac{J-J_{K}^{-}}{J+J_{K}^{-}} \exp +\frac{1}{2 \pi} \int_{-\infty}^{+\infty} \frac{d \tau}{(i \tau-J)} \\
& \cdot\left(\ln D(\tau)+\sum_{K} \ln \left(\frac{i \tau+J_{K}}{i \tau-J_{K}}\right)\right)(\operatorname{Re} J<0), \\
& \operatorname{Det} D_{I I}(J)=F(J) / F_{0}(J) .
\end{aligned}
$$

Moreover, one can derive from (1.12) trace identities that connect integrals of local polynomials in $r, v, w, \ldots$ with the scattering data. The first one reads:

$$
\begin{aligned}
& \frac{1}{2} \int_{0}^{\infty} r\left(v^{2}+2 m v+w^{2}\right) d r \\
& \quad=-\sum_{K}\left(J_{K}^{+}-J_{K}^{-}\right)+\int_{-\infty}^{+\infty} \frac{d \tau}{2 \pi} \ln D(\tau), \text { where }\left\{\begin{array}{l}
\operatorname{Re} J_{K}^{+}>0, \\
\operatorname{Re} J_{K}^{-}<0 .
\end{array}\right.
\end{aligned}
$$

$S_{\text {eff }}$ can finally be reexpressed (Sect. IV) in terms of the scattering data, using the previous results. One gets:

$$
\begin{aligned}
S_{\mathrm{eff}}= & -\frac{1}{4 \pi^{2}} f_{-\infty}^{+\infty} \frac{\ln D(\tau) \ln D\left(\tau^{\prime}\right)}{\left(\tau-\tau^{\prime}\right)^{2}} d \tau d \tau^{\prime}+\sum_{K} \ln \left(\frac{\cos \left(\pi J_{K}\right)}{2 \pi}\right) \\
& -\frac{1}{\pi} \int_{-\infty}^{+\infty}\left(\sum_{K} \frac{\operatorname{sgn} \operatorname{Re} J_{K}}{J_{K}-i \tau}\right) \ln D(\tau) d \tau \\
& -2 \sum_{K \neq K^{\prime}} \operatorname{sgn} \operatorname{Re}\left(J_{K}\right) \operatorname{sgn} \operatorname{Re}\left(J_{K^{\prime}}\right) \ln \left(J_{K}-J_{K^{\prime}}\right) \\
& -\sum_{K} \ln c_{K}+\frac{1}{2 g_{R}} \int_{0}^{+\infty} r w^{2}(r) d r .
\end{aligned}
$$

In the derivation of this equation one uses (1.6) to (1.13) together with a nonstandard trace identity, derived in the Appendix:

$$
\begin{aligned}
Q= & \int_{0}^{+\infty} r \ln m r\left(v^{2}+2 m v+w^{2}\right) d r \\
= & -\frac{1}{4 \pi^{2}} f_{-\infty}^{+\infty} \frac{\ln D(\tau) \ln D\left(\tau^{\prime}\right)}{\left(\tau-\tau^{\prime}\right)^{2}} d \tau d \tau^{\prime} \\
& -\frac{1}{\pi} \int_{-\infty}^{+\infty}\left(\sum_{K} \frac{\operatorname{sgn} \operatorname{Re} J_{K}}{\left(J_{K}-i \tau\right)}\right) \ln D(\tau) d \tau \\
& +\frac{1}{2 \pi} \int_{-\infty}^{+\infty} \ln D(\tau)\left(2 \ln 2+\psi\left(\frac{1}{2}+i \tau\right)+\psi\left(\frac{1}{2}-i \tau\right)\right) d \tau \\
& -2 \ln 2 \sum_{K}\left(\operatorname{sgn} \operatorname{Re} J_{K}\right) J_{K}-\sum_{K}\left(\operatorname{sgn} \operatorname{Re} J_{K}\right) \ln \Gamma\left(\frac{1}{2}+J_{K}\right) \\
& -2 \sum_{K \neq K^{\prime}} \operatorname{sgn}\left(\operatorname{Re} J_{K}\right) \operatorname{sgn}\left(\operatorname{Re} J_{K^{\prime}}\right) \ln \left(J_{K}-J_{K^{\prime}}\right)-\sum_{K} \ln c_{K} .
\end{aligned}
$$


This expression arises owing to renormalization effects in the term $\sim \Gamma\left(1-\frac{v}{2}\right)$ $\cdot \int d^{v} x\left(v^{2}+2 m v+w^{2}\right)$ for $v \rightarrow 2$. Note that a similar identity was found in the bosonic case [5]. This remarkable relation should be linked to an invariance of the linear problem.

The expression (1.14) has a closed form either when $w \equiv 0$, or when $\frac{1}{g_{R}} \equiv 0$, which respectively correspond to Gross-Neveu and Chiral Gross-Neveu, that are known to be integrable in 2 dimensions [11,13-16]. We find again the uppermentioned correspondence between classical and quantum integrability and separateness of the action expressed as a function of the scattering data. The study of instantons gives the following results. The $K^{\text {th }}$-order coefficient of the $\frac{1}{N}$ expansion of any physical quantity behaves as $[2-6,10]$ :

$$
A_{K}=\mathbb{C} \frac{\Gamma\left(K+N_{0} / 2\right)}{\left(-S_{0}\right)^{K+N_{0} / 2}}(1+\mathcal{O}(1 / K)),
$$

where

$S_{0}$ is the effective action of the instanton,

$N_{0}$ is the number of zero modes,

$\mathbb{C}$ is the determinant of small fluctuations around the instanton.

Integrable models do not exhibit any instanton; this can be matched with the fact that in both cases (Gross-Neveu and Chiral Gross-Neveu), the $1 / N$ expansion is known to be convergent for the $S$-matrix [11,13-16] and form factors [15]. As was the case for the non-linear $\sigma$-model [9], this suggests that the $1 / N$ series can be convergent for the Gross-Neveu and CGN models. When $g_{R} \rightarrow-\infty$, a complex action instanton appears, with an effective action $S_{0} \sim-\ln |g|$; the $1 / N$ expansion is simply Borel-summable when one perturbes the Chiral Gross-Neveu model with such a small non-integrable term. Moreover, when $g_{R} \rightarrow 0^{-}$, one finds again that the effective action of the instanton is dominated by a complex term $\frac{1}{g_{R}} \int r w^{2}(r) d r$. In this case, too, the $1 / N$ expansion is Borel-summable. For $g_{R}>0$, the $1 / N$ series is also Borel-summable, but this case is not really interesting since tachyons are present. In contrast with what happened in the bosonic models, there is no clear relation between the limit $g_{R} \rightarrow 0^{-}$of the saddle-point Eqs. (1.5) and the classical fermion field equations.

The general conclusion is that a special role seems to be assigned to the two integrable models (Gross-Neveu and Chiral Gross-Neveu), since they seem to be the only fermionic self-coupled models of the type studied that admit a possibly convergent $1 / N$ expansion.

\section{The Model}

We shall consider in this paper the following $\mathrm{U}(N)$-symmetric and classically scale invariant self-coupled fermionic model:

$$
\mathscr{L}_{0}=\bar{\Psi} \not \Psi+\frac{\lambda_{1}}{2 N}(\bar{\Psi} \Psi)^{2}-\frac{\lambda_{2}}{2 N}\left(\bar{\Psi} \gamma_{5} \Psi\right)^{2} .
$$


This is not the most general Lorentz-invariant fermionic Lagrangian [17]. Vectorvector couplings of the type $\left(\bar{\Psi} \gamma_{\mu} \Psi\right)^{2}$ can also occur in self-coupled theories. We shall not discuss such couplings in this article. In (2.1), $\Psi$ is a $N$-component field $\left(\Psi_{1} \ldots \Psi_{N}\right)$, where each $\Psi_{a}(a=1 \ldots N)$ is a Dirac field in euclidean space. Note that one gets from (2.1) the Gross-Neveu model when $\lambda_{2}=0$, and the Chiral GrossNeveu model when $\lambda_{1}=\lambda_{2}[18]$.

In order to use dimensional regularization [19], we shall introduce Dirac matrices $\gamma_{\mu}^{(v)}$ in any number $v$ of (assumed even) dimensions. These matrices satisfy a Clifford algebra:

$$
\left\{\gamma_{\mu}^{(v)}, \gamma_{\varrho}^{(v)}\right\}=2 \delta_{\mu \varrho}, \quad 1 \leqq \mu, \varrho \leqq v
$$

and act on a $2^{v / 2}$-dimensional spinor space. A well-known and useful recurrent construction reads [20]

$$
\begin{aligned}
& \gamma_{1}^{(v)}=\gamma_{1}^{(2)} \otimes \mathbb{1}^{(v-2)}, \quad \gamma_{2}^{(v)}=\gamma_{2}^{(2)} \otimes \mathbb{1}^{(v-2)}, \\
& \gamma_{\mu}^{(v)}=\gamma_{5}^{(2)} \otimes \gamma_{\mu-2}^{(v-2)} \quad(2<\mu \leqq v) .
\end{aligned}
$$

Here the subscript is a Lorentz index; the superscript indicates the dimension of euclidean space on which the spinorial representation is built.

One can easily check on (2.3) that all trace properties and anticommutation relations of $\gamma^{(v)}$-matrices are verified, provided that the relations are verified for $\gamma^{(v-2)}$ (recurrent construction of $\gamma$-matrices):

$$
\begin{gathered}
\left\{\gamma_{\mu}^{(v)}, \gamma_{\varrho}^{(v)}\right\}=2 \delta_{\mu \varrho}, \\
\operatorname{Tr}\left(\gamma_{\mu}^{(v)}\right)=0, \quad \operatorname{Tr}\left(\gamma_{\mu}^{(v)} \gamma_{\varrho}^{(v)}\right)=\delta_{\mu \varrho} 2^{v / 2}, \\
\operatorname{Tr}[\text { odd number }]=0 .
\end{gathered}
$$

The $\gamma_{1}^{(2)}$ and $\gamma_{2}^{(2)}$ are Pauli matrices, respectively: $\sigma_{1}$ and $\sigma_{2} ; \gamma_{5}^{(2)}$ is the $\sigma_{3}$-matrix.

We also need to define $\gamma_{5}^{(v)}$. This is more difficult: it is well known that dimensional extension of $\gamma_{5}$ is linked to subtle problems, such as the triangle anomaly in spinorial QCD [19]. For later convenience, we define:

$$
\gamma_{5}^{(v)}=\gamma_{5}^{(2)} \otimes \mathbb{1}^{(v-2)} \equiv \gamma_{1}^{(v)} \gamma_{2}^{(v)}
$$

We shall see that this definition is appropriate to dimensional regularization of a two-dimensional theory, and especially to the reduction of a $v$-dimensional-space Dirac equation to a 2-dimensional-space one. However, chiral invariance is partially lost for $v \neq 2$. Moreover, non-standard relations will appear. Namely:

$$
\left\{\gamma_{5}^{(v)}, \gamma_{\mu}^{(v)}\right\}_{+}=0 \quad(1 \leqq \mu \leqq 2), \quad\left[\gamma_{5}^{(v)}, \gamma_{\mu}^{(v)}\right]_{-}=0 \quad(2<\mu \leqq v) .
$$

Trace properties remain. It is easy to check that [omitting the superscript $(v)$ for simplicity]:

$$
\operatorname{Tr} \gamma_{5}=0, \quad \operatorname{Tr} \gamma_{\mu} \gamma_{5}=0, \quad \operatorname{Tr}\left(\gamma_{5} \gamma_{\mu} \gamma_{\varrho} \gamma_{\lambda}\right)=0,
$$

and of course,

$$
\left(\gamma_{5}\right)^{2}=\mathbb{1}^{(v)}
$$


We shall later comment on the consequences of the commutation relations (2.5). The generating functional of the model reads:

$$
\mathscr{Z}=\iint \prod_{a} \mathscr{D} \Psi_{a} \mathscr{D} \bar{\Psi}_{a} \exp \int_{-\infty}^{+\infty} d^{v} x \cdot\left[-\bar{\Psi} \not \Psi-\frac{\lambda_{1}}{2 N}(\bar{\Psi} \Psi)^{2}+\frac{\lambda_{2}}{2 N}\left(\bar{\Psi} \gamma_{5} \Psi\right)^{2}\right] .
$$

A counterterm of the form $\left(\bar{\Psi}_{\gamma_{\mu}} \Psi\right)^{2}$ will also appear in the exponent (2.7). However, this term, which is generated by renormalization effects on the theory defined by (2.1), will be suppressed by an order $(1 / N)$ with respect to the other terms in (2.7). Therefore, it will not appear in the saddle-point equations, and will only contribute to small fluctuation effects around the extrema.

Source terms have also been omitted in (2.7). It will be easy to reintroduce them, when one computes a given Green function. They are not necessary as long as we discuss saddle-points. The $\frac{1}{N}$ expansion of the theory now follows by introducing auxiliary field variables $v$ and $w$ through the Hubbard-Stratonovitch transform [21],

$$
\begin{aligned}
& \exp -\frac{\lambda_{1}}{N} \int_{-\infty}^{+\infty} d^{v} x(\bar{\Psi} \Psi)^{2}=\iint \mathscr{D} v \exp \int_{-\infty}^{+\infty} d^{v} x\left[\frac{N v^{2}}{\lambda_{1}}+2 v \bar{\Psi} \Psi\right], \\
& \exp \frac{\lambda_{2}}{N} \int_{-\infty}^{+\infty} d^{v} x\left(\bar{\Psi} \gamma_{5} \Psi\right)^{2}=\iint \mathscr{D} w \exp \int_{-\infty}^{+\infty} d^{v} x\left[\frac{N w^{2}}{\lambda_{2}}+2 i w \bar{\Psi} \gamma_{5} \Psi\right] .
\end{aligned}
$$

Inserting (2.8) into (2.7) and integrating over fermionic variables leads to the generating functional:

$$
\begin{aligned}
\mathscr{Z}= & \iint \mathscr{D} v \mathscr{D} w \exp N\left[\log \operatorname{det}_{(v)}\left(\phi-v-i \gamma_{5} w\right)+\frac{1}{2 \lambda_{1}} \int_{-\infty}^{+\infty} d^{v} x v^{2}(x)\right. \\
& \left.+\frac{1}{2 \lambda_{2}} \int_{-\infty}^{+\infty} d^{v} x w^{2}(x)\right] .
\end{aligned}
$$

It is clear from expression (2.9) that we have obtained an effective (non-local) theory with $1 / N$ as coupling constant. The action of this theory has the well-known structure of a "1-loop-effective action," introduced in [1], namely the ln det of a differential operator containing the fields, plus local terms in the fields. We have already met with such effective actions when we were studying $1 / N$ expansion of bosonic theories $V\left(\Phi^{2}\right)$ and $\left(\Phi^{2}\right)^{2}[2-9]$. Owing to the fermionic nature of the initial field variables, the $\ln$ det now exhibits a + sign (instead of the - sign of bosonic $1 / N$ theories).

Let us now proceed as usual and look for constant saddle-points. One gets in the present case:

$$
\operatorname{tr}\left\langle x\left|\frac{1}{\phi-v-i \gamma_{5} w}\right| x\right\rangle=\frac{v}{\lambda_{1}}, \quad \operatorname{tr}\left\langle x\left|\frac{i \gamma_{5}}{\not-v-i \gamma_{5} w}\right| x\right\rangle=\frac{w}{\lambda_{2}},
$$

where "tr" means taking the trace over spinor indices. Constant solutions of (2.10) can be found in general by computing exactly the constant field propagator in (2.10). We shall study the vacuum structure of these fermionic models, using 

another regularization. Dimensional extension of $\gamma_{5}$ according to $\gamma_{5}=\prod_{i=1}^{v} \gamma_{i}$ (for $v$
even) is such that

$$
\left\{\gamma_{5}, \gamma_{\mu}\right\}=0, \quad\left(\gamma_{5}\right)^{2}=\mathbb{1}, \quad \operatorname{Tr} \gamma_{5}=0, \quad \operatorname{Tr}\left(\gamma_{5} \gamma_{\mu}\right)=0,
$$

for all $1 \leqq \mu \leqq v$. Equation (2.10) can be rewritten now as:

$$
\begin{aligned}
& -\frac{2^{v / 2} \Gamma(1-v / 2)}{(4 \pi)^{v / 2}} \cdot v \cdot\left(v^{2}+w^{2}\right)^{\frac{v-2}{2}}=\frac{v}{g_{1}} \quad\left(\lambda_{1} \equiv g_{1}\right), \\
& \frac{2^{v / 2} \Gamma(1-v / 2)}{(4 \pi)^{v / 2}} \cdot w \cdot\left(v^{2}+w^{2}\right)^{\frac{v-2}{2}}=\frac{w}{g_{2}} \quad\left(\lambda_{2} \equiv g_{2}\right) .
\end{aligned}
$$

In the general case when $g_{1}$ is different from $g_{2}$ (other models than Chiral GrossNeveu), these saddle-point equations only have two possible solutions: either $v=0, w=m$, which leads to a renormalization of $g_{2}$ according to:

$$
\frac{1}{g_{2}}=\frac{2^{v / 2} \Gamma(1-v / 2)}{(4 \pi)^{v / 2}} m^{v-2}
$$

or $v=m, w=0$, with a corresponding renormalization of $g_{1}$. Note that $v$ and $w$ have a physical meaning, whichever regularization scheme is chosen, since the propagator of fermions, to leading order in $1 / N$, reads:

$$
G(x, y)=\left\langle x\left|\frac{1}{\not+v_{0}+i \gamma_{5} w_{0}}\right| y\right\rangle .
$$

$m$ is the dynamically generated mass (to leading order in $1 / N$ ) of the physical $N$-plet of fermions. Actually, these two saddle-points generate equivalent theories: through a finite chiral rotation $\Psi \rightarrow e^{i \frac{\pi}{4} \gamma_{5}} \Psi$, one can see that $v$ is exchanged with $w$ in the functional determinant. Hence the $1 / N$ expansion around any of the two vacua is the same, provided that one also exchanges the role of $g_{1}$ and $g_{2}$; moreover, the non-constant saddle-points of the renormalized action, $v_{c}, w_{c}$, corresponding to the two possible choices of background configuration, will be obtained one from the other by exchanging $v_{c}$ and $w_{c}$. It is therefore enough to study one of these two theories. We shall use $v=m, w=0$ as the background configuration for instantons, since the mass term is here more natural than the " $\gamma_{5}$ " mass corresponding to $w=m, v=0$.

In the case of the Chiral-Gross-Neveu model, (2.11) reduces to a single equation: any couple of values $v$ and $w$ such that $v^{2}+w^{2}=m^{2}$ is a possible vacuum; $m$ is again the physical mass of the $N$-plet of fermions, and $g_{1}=g_{2}$ is renormalized according to

$$
\frac{1}{g}=-\frac{2^{v / 2} \Gamma(1-v / 2)}{(4 \pi)^{v / 2}} m^{v-2} .
$$

This degeneracy is due to the chiral invariance of the theory. Now if one does a careless expansion around one of those degenerate vacuum solutions (for instance $v=m, w=0$ as in [22]), one gets infrared divergences owing to this illegal breaking of chiral invariance in two dimensions [23]. Perturbation theory (here $1 / N$ 
expansion) must be done according to [14] or [16]: one extracts from the initial fermionic field $\Psi$ a massless field, corresponding to local chiral transformations that leave $S$ invariant; this massless field compensates the infra-red divergences of the theory. This procedure can also be done in the framework of path integral method through a change of variables in the integral [24]. However, only the gradient of the chiral phase of $v+i \gamma_{5} w$ is the relevant massless field. One can then choose the absolute chiral phase of the vacuum configuration. We shall set again $v=m, w=0$. Of course, if we want to do a perturbative expansion of the functional integral around non-constant saddle-points, we should be very careful about the treatment of such IR problems. Anyway, the conclusion is that, as long as we are looking for saddle-points, we can choose the vacuum configuration $v=m$, $w=0$ as a background for the instantons, without any problem, for all the quarticcoupled fermionic theories that we are studying.

Let us now come back to our non-chirally-invariant regularization $\gamma_{5}=\gamma_{1} \gamma_{2}$. The previous choice of vacuum $v=m, w=0$ still holds as a solution of (2.10), as can easily be seen by computing directly $\left\langle x\left|\frac{1}{\phi+v}\right| x\right\rangle$. Again, $g_{1}$ is renormalized
according to:

$$
\frac{1}{g_{1}}=-\frac{2^{v / 2} \Gamma(1-v / 2)}{(4 \pi)^{v / 2}} v_{0}^{v-2},
$$

and again $v_{0} \equiv m$ is a dynamically generated mass for the $N$-plet of fermions. As is clear, the physical results, including non-constant saddle-points, and the large order behaviour of $1 / N$ expansion for physical quantities, controlled by these saddle-points, will not depend on the regularization scheme, as long as the independent parameters (mass spectrum ...) are the same in the end, as is the case here for the fermion mass. We shall therefore keep from now on this non-chirally invariant regularization scheme, which is much easier to deal with, as we are going to see, when one looks for non-constant saddle-points.

Let us make a few comments about Eq. (2.15). We find the well-known mass generation of the quantum theory in first order of the $1 / N$ expansion [18]. From now on we shall set $v_{0} \equiv m$. Indeed, it is clear that at first order in $1 / N$ the spectrum of the theory contains $N$ massive fermions. Moreover, Eq. (2.11) clearly provides dimensional transmutation in $v=2$ dimensions. The coupling constant $g_{1}$, which is dimensionless, is replaced after renormalization through (2.11), by a dimensionful parameter $m$. When $g_{1}$ and $g_{2}$ are independent (neither G.N. nor C.G.N.), a dimensionless parameter $g_{2_{R}}$ [see (2.17)] will remain in the quantum theory. We now rewrite the effective action in (2.9) in term of the renormalized parameters, and shifted fields $v \rightarrow v-v_{0}, w \rightarrow w$. We assume from now on that $v$ and $w$ are a priori complex functions. $S_{\text {eff }}$ reads

$$
\begin{aligned}
S_{\text {eff }}= & \log \operatorname{det}_{(v)}\left(\frac{\not-m-v-i \gamma_{5} w}{\not \supset-m}\right)-2^{v / 2} \frac{m^{v-2}}{(4 \pi)^{v / 2}} \Gamma(1-v / 2) \\
& \cdot \int_{-\infty}^{+\infty} d^{v} x\left(v^{2}+2 m v+w^{2}\right)+\frac{1}{g_{2_{R}}} \int_{-\infty}^{+\infty} d^{v} x w^{2}(x) .
\end{aligned}
$$


As already mentioned, our choice of $\gamma_{5}$ restricts chiral invariance of the theory to two dimensions. An alternative "recurrent" construction $\gamma_{5}^{(v)}=\prod_{\mu=1}^{v} \gamma_{\mu}^{(v)}$ would preserve complete chiral invariance, but as we shall see afterwards, it would make the mechanism of dimensional regularization of the Log det more difficult to handle for non-constant fields.

It is necessary to make a comment on the expression (2.16). Equation (2.11) insures that $\frac{\delta S}{\delta v}=\frac{\delta S}{\delta w}=0$ when the shifted potentials $v$ and $w$ are set equal to zero. Equation (2.11) only implies a renormalization of $g_{1}$. However, one can see that the ln det has two divergences: one is the term linear + quadratic in $v$ which is exactly cancelled when (2.11) is satisfied, and $v$ is shifted as $v \rightarrow m+v$, the other is purely quadratic in $w$. This last divergence will be cancelled by a renormalization of $g_{2}$ according to:

$$
\frac{1}{g_{2_{B}}}=\frac{1}{g_{1_{B}}}+\frac{1}{g_{2_{R}}}
$$

( $B$ for bare, $R$ for renormalized). (The Chiral Gross-Neveu model corresponds to $1 / g_{R} \equiv 0$.) One can then show straightforwardly that $S_{\text {eff }}$ in (2.12) is indeed finite. As we explained in the beginning, no term other than those already existing in $S_{\text {eff }}$ will arise through renormalization, at least at order $N$. The theory defined with only scalar and pseudo-scalar coupling can be renormalized at leading order in $N$, and therefore the computation of saddle-points and the qualitative conclusions that we shall draw on the large order behaviour of the $1 / N$ expansion will be correct (see [10] for a discussion on saddle-points + large orders of a renormalized field theory). An interesting feature arises when one does the computation of $S_{\text {eff }}$ by systematically expanding the $\ln \operatorname{det}$ in powers of $\left(\frac{v+i \gamma_{5} w}{\not \partial-m}\right)$. Owing to the commutation of $\gamma_{s}^{(v)}$ with the $\gamma_{\mu}^{(v)}$ for $\mu>2$, we must separate $\left(p_{1}, p_{2}\right)$ and $\left(p_{\mu}\right), \mu>2$, when integrating. The use of commuting or non-commuting $\gamma_{5}^{(v)}$ matrix leads to a regularized (ln det) differing by a (const) times $\int_{-\infty}^{+\infty} w^{2}(x) d^{v} x,+\mathcal{O}(v-2)$.

This effect, that follows from the aforesaid dimensional extension of $\gamma_{5}$, arises therefore in the same way as the triangle anomaly in spinor electrodynamics [19].

We shall now analyse the particle spectrum in the large $N$ limit by computing the small fluctuations around the saddle-points $v=0, w=0$ of the effective action (2.16). We obtain the following inverse propagators for $v$ and $w$ fields $(v=2)$ :

$$
\begin{gathered}
\left.\frac{\delta^{2} S}{\delta v_{(x)} \delta v_{(y)}}\right|_{\substack{w=0 \\
v=0}}=\int \frac{d^{2} p}{(2 \pi)^{2}} e^{i p(x-y)} \Delta_{v}^{-1}(p), \\
\left.\frac{\delta^{2} S}{\delta w_{(x)} \delta w_{(y)}}\right|_{\substack{w=0 \\
v=0}}=\int \frac{d^{2} p}{(2 \pi)^{2}} e^{i p(x-y)} \Delta_{w}^{-1}(p), \\
\left.\frac{\delta^{2} S}{\delta v_{(x)} \delta w_{(y)}}\right|_{\substack{w=0 \\
v=0}}=0 .
\end{gathered}
$$


Here:

$$
\begin{gathered}
\Delta_{v}^{-1}(p)=-\frac{1}{\pi} \sqrt{1+\frac{4 m^{2}}{p^{2}}} \cdot \operatorname{Arg} \tanh \frac{1}{\sqrt{1+\frac{4 m^{2}}{p^{2}}}}, \quad\left(p^{2}+4 m^{2}\right) p^{2}>0, \\
\Delta_{w}^{-1}(p)=\frac{1}{g_{R}}-\frac{1}{\pi \sqrt{1+\frac{4 m^{2}}{p^{2}}}} \operatorname{Arg} \tanh \frac{1}{\sqrt{1+\frac{4 m^{2}}{p^{2}}}} .
\end{gathered}
$$

Apart from the supplementary term $\frac{1}{g_{R}}$ in $\Delta_{w}^{-1}(p)$, one finds here the propagators obtained in [22] when "naively" computing the $1 / N$ expansion for Chiral GrossNeveu around the vacuum $v=m, w=0$.

It appears in (2.19) that if $g_{R}>0, \Delta_{w}(p)$ exhibits a pole for some $p^{2}=m_{T}^{2}>0$. This corresponds to a tachyon in the $w$ channel. So we have to set $g_{R}<0$. In this case, one finds a pole in $\Delta_{w}(p)$ at some $p^{2}=-m_{B}^{2}<0$, where $m_{B}\left(g_{R}\right)$ is the real positive solution of the equation

$$
\frac{1}{g_{R}}+\frac{1}{\pi} \frac{1}{\sqrt{\frac{4 m^{2}}{M_{B}^{2}}-1}}=\operatorname{Arctan} \frac{1}{\sqrt{\frac{4 m^{2}}{M_{B}^{2}}-1}}=0 .
$$

One finds $4 m^{2}>M_{B}^{2}(g)>0$ for $0>g_{R}>-\infty$. We can therefore interpret this physical pole in the $w$-channel as a fermion-antifermion bound state. (Remember that $w$ is associated to $\bar{\Psi} \gamma_{5} \Psi$.) It can easily be checked that the residue of $\Delta_{w}$ has the correct sign, so that this pole does not correspond to a ghost. This allows a physical renormalization-invariant interpretation of $g_{R}$ as being a function of the two independent masses in the theory. The propagator $\Delta_{v}(p)$ has no poles. Both propagators have a cut for $p^{2}<-4 m^{2}$, corresponding to two-particle production. Finally, we note that in the CGN limit, $1 / g_{R} \rightarrow 0$, and the $w$-bound state becomes massless. This is the well-known "naive" result of infra-red divergence in the $1 / N$ expansion of CGN, and can be cured (as explained before) by a careful treatment of this infra-particle [11]. We will now compute the functional determinant in (2.16). The structure and recurrent construction of $\gamma_{\mu}$ and $\gamma_{5}$ matrices will be very useful in this computation. We choose $v$ and $w$ to depend only on the two variables $x_{1}$ and $x_{2}$; we shall later on reduce it to a spherically-symmetric dependence on $r=\sqrt{x_{1}^{2}+x_{2}^{2}}$. Taking a $v$-dimensional spherically symmetric ansatz would lead to a more involved regularization procedure. The eigenvalue problem reads:

where

$$
\mathscr{D} \Psi=\mathscr{E} \Psi,
$$

$$
\begin{aligned}
\mathscr{D}= & \not \phi^{(v)}-m-v-i \gamma_{5}^{(v)} w \\
= & \left(\sigma_{1} \partial_{1}+\sigma_{2} \partial_{2}\right)^{(2)} \otimes \mathbb{1}^{(v-2)}+\sigma_{3} \otimes \not^{(v-2)} \\
& -m \mathbb{1}^{(v)}-v \mathbb{1}^{(v)}-i w\left(\sigma_{3} \otimes \mathbb{1}^{(v-2)}\right) \\
= & {\left[\begin{array}{cc}
(\not-(m+v+i w)) \mathbb{1}^{(v-2)} & \left(\partial_{x}+i \partial_{y}\right) \mathbb{1}^{(v-2)} \\
\left(\partial_{x}-i \partial_{y}\right) \mathbb{1}^{(v-2)} & -\not-(m+v-i w) \mathbb{1}^{(v-2)}
\end{array}\right] . }
\end{aligned}
$$


The elements of the $2 \times 2$ matrix (2.21) are operators acting on a $2^{v / 2-1}$ dimensional space. $2^{v / 2}$-dimensional spinors have been decomposed into "upper" and "lower" components, each taking value in a $2^{v / 2-1}$-dimensional space. The eigenfunctions will be the combination of such "upper" and "lower" $2^{v / 2-1}$. dimensional spinors.

Using the cyclic symmetry of the $x^{\lambda}(\lambda>2)$ variables enables one to conclude that eigenspinors can be chosen with a well-defined transverse momentum $\left(K^{\lambda}\right.$, $\lambda>2)$.

$$
\Psi=\left(\begin{array}{l}
\Psi_{1}\left(x_{1}, x_{2}\right) \\
\Psi_{2}\left(x_{1}, x_{2}\right)
\end{array}\right) e^{i \hat{K} x}
$$

where

$$
\hat{K}_{1}=\hat{K}_{2}=0 \text {. }
$$

It is now possible to expand $\Psi_{1}$ and $\Psi_{2}$ on a base of eigenspinors of $\hat{K}$. This is made possible by the recurrent construction of $\gamma_{\mu}$-matrices: it insures that the "supplementary" operator in $\not \phi^{(v)}$ is $\gamma_{5}^{(2)} \otimes \not^{(v-2)}$, where $\not^{(v-2)}$ is a complete free Dirac-equation operator acting on a $2^{v / 2-1}$-spinor space, and that the matrices $\gamma_{1}^{(v)}$, $\gamma_{2}^{(v)}$, and $\gamma_{5}^{(v)}$ act trivially on the "upper" and "lower" subspace. The eigenvalue equation now reads:

$$
\left.\begin{array}{rl}
{[i \hat{K}-(m+v+i w)] \Psi_{1}+\left[\partial_{x}+i \partial_{y}\right] \Psi_{2}} & =\mathscr{E} \Psi_{1} \\
{\left[\partial_{x}-i \partial_{y}\right] \Psi_{1}+[-i K-(m+v-i w)] \Psi_{2}} & =\mathscr{E} \Psi_{2}
\end{array}\right\} .
$$

Expanding now $\Psi_{1}$ and $\Psi_{2}$ in a base of eigenspinors of $\hat{K}$, and projecting the eigenvalue Eq. (2.23) on this base lead to the following equations:

$$
\left.\begin{array}{r}
{[i \lambda-(m+v+i w)] f_{1}+\left[\partial_{x}+i \partial_{y}\right] f_{2}=\mathscr{E} f_{1}} \\
{\left[\partial_{x}-i \partial_{y}\right] f_{1}+[-i \lambda-(m+v-i w)] f_{2}=\mathscr{E} f_{2}}
\end{array}\right\},
$$

where $\lambda$ is the eigenvalue of $\hat{K}$. $\lambda$ can take the two values $\pm \sqrt{\hat{K}^{2}}$. Hence the eigenvalues $\mathscr{E}$ have an intrinsic degeneracy $2^{v / 2-2}$, associated to the degeneracy of a free Dirac eigenspinor, in $(v-2)$ spatial dimensions, with a specified eigenvalue $\lambda$.

Again we would like to emphasize that this sort of "dimensional reduction" of (2.22) to (2.24) heavily relies on the recurrent tensor-product construction of $\gamma_{\mu}^{(v)}$ and on the definition $\gamma_{5}^{(v)}=\gamma_{5}^{(2)} \otimes \mathbb{1}^{(v-2)}$. Otherwise (if we had for instance chosen a recurrent definition $\left.\gamma_{5}^{(v)}=\prod_{\mu=1}^{v} \gamma_{\mu}^{(v)}\right)$, we would have had to deal explicitly with those $2^{v / 2-1}$-dimensional spinors which we have introduced before.

It now becomes easy to express the determinant in (2.16) in terms of the twodimensional operator $D_{I I}(\lambda)$ that appears in (2.24). We have:

$$
\log \operatorname{det} \mathscr{D}_{(v)}=\left(\frac{L}{2 \pi}\right)^{v-2} 2^{v / 2-2} \cdot \int^{ \pm} d^{v-2} \hat{K} \log \operatorname{det} D_{I I}(\lambda), \quad \lambda= \pm \sqrt{\hat{K}^{2}}
$$

Let us make a few remarks concerning this formula:

- the $(L / 2 \pi)^{v-2}$ comes from partial Fourier-transform that changes the variables $x_{\mu}, \mu>2$, into $\hat{K}_{\mu}, \mu>2$, when computing the tr $\log \mathscr{D}_{(v)}$; integration over $d^{v-2} x$, when computing $\int_{-\infty}^{+\infty}\left\langle x\left|\log \mathscr{D}_{(v)}\right| x\right\rangle d^{v} x$, generates the $(L / 2 \pi)^{v-2}$ factor; 
- the factor $2^{v / 2-2}$ is the "intrinsic degeneracy" of any eigenvalue $\mathscr{E}$ associated to a given $\lambda$;

- the $\int^{ \pm} d^{v-2} \hat{K}$ means that we have not only to integrate over the length and direction of transverse momentum $\hat{K}$, but also to take into account the two possible signs of the eigenvalue $\lambda$ in (2.24). In fact, this is a correct expression for $\sum_{(\lambda)} \ln \operatorname{det} D_{I I}(\lambda)$

Assuming now that $v$ and $w$ are 2-dimensional rotationally invariant, enables one to compute $\log \operatorname{det} D_{I I}(\lambda)$. First of all we shall recast it into a simpler form, using the rotational invariance of $v(r)$ and $w(r)$. We introduce the angular momentum operator in 2 dimensions acting on spinors: $\mathscr{I}=+i \partial_{\theta}+\frac{\sigma_{3}}{2}$, where polar coordinates are introduced: $x_{1}=r \cos \theta ; x_{2} \equiv-r \sin \theta$. This operator clearly commutes with $D_{I I}$. The eigenvectors of $D_{I I}$ can therefore be chosen as eigenvectors of $\mathscr{I}$. Namely:

$$
\Psi(r, \theta, J)=e^{-i J \theta}\left(\begin{array}{c}
\varphi_{+}(r) e^{+i \theta / 2} \\
\varphi_{-}(r) e^{-i \theta / 2}
\end{array}\right) .
$$

The logarithm of the determinant of $D_{I I}$ will then read as a sum of logarithms of determinants in each partial wave sector. $D_{I I}(J)$ reads then:

$$
D_{I I}(J)=\left(\begin{array}{cc}
-m-v-i w+i \lambda & d_{r}+\frac{J+\frac{1}{2}}{r} \\
d_{r}-\frac{J-\frac{1}{2}}{r} & -m-v+i w-i \lambda
\end{array}\right) .
$$

We can now rewrite (2.26) as:

$$
\ln \operatorname{det} \mathscr{D}_{v}=\left(\frac{L}{2 \pi}\right)^{v-2} \cdot 2^{v / 2-2} \cdot \int^{ \pm} d^{v-2} \lambda\left(\sum_{J=-\infty+1 / 2}^{J=+\infty+1 / 2} \ln \operatorname{det} D_{I I}(J)\right) .
$$

The eigenvalues $J$ are taken to be half plus integer, so that $\Psi(r, \theta+2 \pi, J)$ $=\Psi(r, \theta, J)$. This is compatible with the fermionic nature of the eigenvectors (i.e. half plus integer angular momentum). No degeneracy arises due to $J$, since angular momentum is not degenerate in 2 dimensions ( $J$ can be positive or negative).

Expression (2.28) holds for any two-dimensional rotationally invariant potentials $v$ and $w$. This restriction is not too serious, since our final purpose is to compute large orders in $1 / N$ by evaluating extremal action configurations. The dominant instanton usually exhibits maximal symmetry (note that this was proved [12] rigorously in the case of a local euclidean action).

We shall now express the complete effective action (2.16) in a partial wave expansion. We need therefore to obtain such an expansion for the local term $\sim \Gamma(1-v / 2)$. This will be provided by the following remark. The linear part of ln $\operatorname{det} \mathscr{D}(v)$ in $v$ reads

$$
\ln \operatorname{det} \frac{\mathscr{D}(v, \lambda)}{\mathscr{D}_{0}(v, \lambda)}=2^{v / 2} \frac{m^{v-2}}{(4 \pi)^{v / 2}} \Gamma(1-v / 2) \int_{-\infty}^{+\infty} d^{v} x v(x)+\mathcal{O}\left(v^{2}, w^{2}\right) .
$$


We can also obtain this linear part from (2.28), which gives:

$\ln \operatorname{det} \frac{\mathscr{D}(v, \lambda)}{\mathscr{D}_{0}(v, \lambda)}$

$$
\begin{aligned}
= & 2^{v / 2-1} L^{\nu-2} \int^{( \pm)} \frac{d^{v-2} \lambda}{(2 \pi)^{v-2}} \int_{0}^{+\infty} \sum_{J>0}^{+\infty+1 / 2}\left[\left(I_{J+1 / 2} K_{J+1 / 2}+I_{J-1 / 2} K_{J-1 / 2}\right)\left(r \sqrt{\lambda^{2}+m^{2}}\right)\right] \\
& \cdot m r v(r) d r+\mathcal{O}\left(v^{2}, w^{2}\right) .
\end{aligned}
$$

Equation (2.24) follows from expanding $\ln \operatorname{det} \frac{D(J, \lambda)}{D_{0}(J, \lambda)}$ as:

$$
\ln \operatorname{det} \frac{D(J, \lambda)}{D_{0}(J, \lambda)}=\operatorname{tr} \int_{0}^{+\infty} v(r) d r G_{J}(r, r, \lambda)+\mathcal{O}\left(v^{2}, w^{2}\right) .
$$

Here $G_{J}\left(r, r^{\prime}, \lambda\right)$ stands for the Green function of the operator $D_{0}(J, \lambda)$. We have:

$$
\begin{aligned}
& \operatorname{tr} G_{J}\left(r, r^{\prime}, \lambda\right) \\
& =r\left[(m+i \lambda) I_{J-1 / 2}\left(\mu r_{<}\right) K_{J-1 / 2}\left(\mu r_{>}\right)+(m-i \lambda) I_{J+1 / 2}\left(\mu r_{<}\right) K_{J+1 / 2}\left(\mu r_{>}\right)\right],
\end{aligned}
$$

where $\mu=\sqrt{\lambda^{2}+m^{2}}$ and $r_{>}, r_{<}$are respectively the larger and smaller of $r$ and $r^{\prime}$. gives

Matching now (2.30) and (2.29) for a spherically symmetric $v(r)$ immediately

$$
\begin{aligned}
2^{v / 2} & \Gamma(1-v / 2) \cdot \frac{2 \pi}{(4 \pi)^{v / 2}} \\
& =2^{v / 2-2} \int^{ \pm} \frac{d^{v-2} \lambda}{(2 \pi)^{v-2}} \sum_{1 / 2}^{+\infty+1 / 2}\left[\left(I_{J+1 / 2} K_{J+1 / 2}+I_{J-1 / 2} K_{J-1 / 2}\right)\left(\sqrt{\lambda^{2}+m^{2}} r\right)\right]
\end{aligned}
$$

The series in (2.33) is formally divergent. It must be understood everywhere in this paper that such divergent series are regularized as $\lim _{\varphi \rightarrow 0} \sum_{J=0}^{+\infty}\left(I_{J} K_{J}\right) \cos J \varphi$. The
divergence now appears as

$$
\sum_{J=0}^{+\infty} I_{J} K_{J} \cos J \varphi=-\ln \varphi+\text { finite part }
$$

Since the divergent term " $\ln \varphi$ " does not depend on $\lambda$ in (2.33), the dimensionally regularized integral of this term gives 0 , and therefore the integral in (2.30) and (2.33) is well defined once this regularization procedure is assumed. Note that this divergence is not the same as the $\Gamma(1-v / 2)$ divergence in (2.33). This can easily be seen when proving directly (2.33). In fact, the finite part in (2.34) is known to be equal to $-\gamma+2 \ln 2-\ln \left(\sqrt{\lambda^{2}+m^{2}} r\right)$ (this follows from taking the limit of the Gegenbauer sum rule $K_{0}(w)=J_{0} K_{0}+\sum_{J=1}^{+\infty} I_{J} K_{J} \cos J \varphi$, where $w=\sqrt{2 r^{2}} \sqrt{1-\cos \varphi}$, and expanding $K_{0}$ around $w=0$ [25]). It is this part, $\sim \ln \sqrt{\lambda^{2}+m^{2}}$, that gives the divergent behaviour for $v \rightarrow 2$ after integrating over $d^{v-2} \lambda$. Equation (2.33) 
immediately follows from:

$$
2^{v / 2-2} \int^{ \pm} \frac{d^{v-2}}{(2 \pi)^{v-2}} \ln \sqrt{\lambda^{2}+m^{2}}=-\Gamma(1-v / 2) \frac{2^{v / 2}}{(4 \pi)^{v / 2}} \cdot 2 \pi m^{v-2}
$$

It is now possible to replace the counterterm $\Gamma(1-v / 2) \int_{0}^{+\infty}\left(v^{2}+\check{2} m v+w^{2}\right) r d r$ in the effective action $S_{\text {eff }}$ by its "partial wave expansion." The effective action can thus be recast into the form

$$
\begin{aligned}
S_{\mathrm{eff}}= & \frac{L^{v-2}}{(2 \pi)^{\left(\frac{v}{2}-1\right)}} 2^{v / 2-2} \int^{ \pm} d^{v-2} \lambda \\
& \cdot \sum_{\ell=0}^{+\infty}\left(\ln \operatorname{det} D\left(\ell+\frac{1}{2}, \lambda\right)+\ln \operatorname{det} D\left(-\ell-\frac{1}{2}, \lambda\right)\right. \\
& \left.-\int_{0}^{+\infty} r\left(v^{2}+2 m v+w^{2}\right) \cdot\left(I_{\ell}(\mu r) K_{\ell}(\mu r)\right) \cdot\left(2-\delta_{\ell_{0}}\right) d r\right) .
\end{aligned}
$$

As we shall see, the existence and nature of trace identities, together with the finiteness of the series $\sum_{J=1}^{+\infty}\left(I_{J} K_{J}-\frac{1}{2} J\right)$, will imply that the series $\sum_{\ell=0}^{+\infty}(\ldots)$ under the integration sign is convergent [this time without any regularization as in (2.34)].

It becomes now possible to take the limit $v \rightarrow 2$ of the effective action, and as it was to be expected (since the theory is now renormalized), this action is finite. Since the functions appearing in (2.36) under the $\int^{ \pm} d^{v-2} \lambda$ are test-functions, decreasing to 0 when $\lambda$ goes to $+\infty$, we can use the limit:

$$
\int^{ \pm} d^{v-2} \lambda f(\lambda) \underset{v \rightarrow 2}{=} 2 f(0)
$$

The effective action now reads:

$$
\begin{aligned}
S_{\mathrm{eff}}= & \sum_{J=1 / 2}^{+\infty}[\ln \operatorname{det} D(J)+\ln \operatorname{det} D(-J) \\
& \left.-\int_{0}^{+\infty}\left(v^{2}+2 m v+w^{2}\right)\left(I_{J-1 / 2}(m r) K_{J-1 / 2}(m r)\right)\left(2-\delta_{J-1 / 2,0}\right) r d r\right] .
\end{aligned}
$$

Using the equality (2.34) finally gives:

$$
\begin{aligned}
S_{\mathrm{eff}}= & \sum_{J=1 / 2}^{+\infty}\left[\ln \operatorname{det} D(J)+\ln \operatorname{det} D(-J)-\frac{1}{J}\left(\int_{0}^{+\infty} r\left(v^{2}+2 m v+w^{2}\right) d r\right)\right] \\
& +\int_{0}^{+\infty} r \ln m r\left(v^{2}+2 m v+w^{2}\right) d r+(-2 \ln 2+\gamma) \\
& \cdot \int_{0}^{+\infty} r\left(v^{2}+2 m v+w^{2}\right) d r+\frac{1}{2 g_{R}} \int_{-\infty}^{+\infty} r w^{2}(r) d r .
\end{aligned}
$$

\section{Inverse Scattering Transform}

The effective action obtained in Sect. II has a non-local form that makes its direct study rather untractable. It is necessary to introduce new variables, in terms of which $S_{\text {eff }}$ will have a local form. This will enable us to study the saddle-points of 
such effective actions by extremizing $S_{\text {eff }}$ with respect to those new variables, and to draw conclusions about large order behaviour of $1 / N$ expansion, that is known to be dominated by those "instantons" [10]. Note that a rigorous proof of this domination ${ }^{1}$ was recently given for $g\left(\Phi^{2}\right)_{I I}^{2}[26 \mathrm{a}, \mathrm{b}]$ and $g\left(\Phi^{2}\right)_{I I I}^{2}[26 \mathrm{c}]$. The shape of $S_{\text {eff }}$ in (2.30) clearly indicates to us that the new variables which must be introduced are the scattering data associated to the linear problem of Dirac equation in 2 dimensions with rotational symmetry of the potential, and the angular momentum as spectral parameter. Indeed, $S_{\text {eff }}$ appears as the sum of Fredholm radial determinant of Dirac equation plus local terms in the potential.

We shall give now the main features and some useful results for inverse scattering transforms (IST) in Dirac equations, using the angular momentum $J$ as the spectral parameter. Note that the IST for the one-dimensional Dirac equation, using energy as a spectral parameter, has already been considered [27]; we believe that the IST in angular momentum is new; moreover, it allows one to solve interesting field-theory problems. The main characteristics of this IST and the framework of reasoning are the same as for the IST in angular momentum for the Schrödinger equation [7]. The Dirac equation in 2 (regularized) dimensions reads:

$$
\left[\begin{array}{cc}
i \lambda-m-v-i w & e^{i \theta}\left(\partial_{r}+\frac{i}{r} \partial_{\theta}\right) \\
e^{-i \theta}\left(\partial_{r}-\frac{i}{r} \partial_{\theta}\right) & -i \lambda-m-v+i w
\end{array}\right]\left[\begin{array}{l}
\psi^{(1)} \\
\psi^{(2)}
\end{array}\right]=0,
$$

where $v \equiv v(r), w \equiv w(r), v$ and $w$ are complex potentials; $\lambda$ is defined in Sect. II (it corresponds to the degrees of freedom in $v-2$ dimensions); polar coordinates read:

$$
x_{1}=r \cos \theta, \quad x_{2}=-r \sin \theta .
$$

The differential operator (3.1) commutes with the angular momentum

$$
\mathscr{I}=i \partial_{\theta}+\frac{\sigma_{3}}{2}
$$

Denoting by $J$ its eigenvalue, one gets the reduced system:

$$
\left.\begin{array}{l}
\left(d_{r}+\frac{J}{r}\right) \varphi^{(2)}=(-i \lambda+m+v+i w) \varphi^{(1)} \\
\left(d_{r}-\frac{J}{r}\right) \varphi^{(1)}=(i \lambda+m+v-i w) \varphi^{(2)}
\end{array}\right\},
$$

where we have set

$$
\Psi=\left(\begin{array}{l}
\psi^{(1)}=\chi^{(1)}(r) \exp \left(-\frac{1}{2}\left(J-\frac{1}{2}\right) \theta\right) \\
\psi^{(2)}=\chi^{(2)}(r) \exp \left(-\frac{1}{2}\left(J+\frac{1}{2}\right) \theta\right)
\end{array}\right.
$$

and

$$
\varphi^{(1)(2)}(r)=\chi^{(1)(2)}(r) \cdot \sqrt{r \cdot m} .
$$

1 For the perturbation theory in $g$ 
We shall now study the linear problem (3.4) where we consider $J$ as the spectral parameter.

Let us first define the "scalar product" of two spinors. We set:

$$
\left\langle\Phi_{1} \mid \Phi_{2}\right\rangle=\int_{0}^{+\infty} \frac{d r}{r}\left(\Phi_{1}^{(1)}(r) \Phi_{2}^{(2)}(r)+\Phi_{1}^{(2)}(r) \Phi_{2}^{(1)}(r)\right) .
$$

This "scalar product" is neither hermitian, nor positive definite. However, this definition implies that the differential operator $D$ is symmetric with respect to (3.6). Moreover, we shall see that amongst the scattering data, a term appears which is the normalization coefficient of the eigenfunction $\left(\Phi_{K}\right)$ with respect to this "scalar product." We shall now define the Jost solutions and the Jost functions, which are the fundamental objects of this study. The Jost solutions are defined as (regular solutions for $r \rightarrow 0$ ):

$$
\begin{aligned}
& \operatorname{Re} J>0: \quad f^{+}(J) \underset{(r \rightarrow 0)}{=}\left\{\begin{array}{l}
\mu^{J} r^{J}, \\
\mu^{J}\left(\frac{-i \lambda+m+v+i w(0)}{2 J+1}\right) r^{J+1},
\end{array}\right. \\
& \operatorname{Re} J<0: \quad f^{-}(J) \underset{(r \rightarrow 0)}{=}\left\{\begin{array}{l}
\mu^{-J} r^{-J+1}\left(\frac{i \lambda+m+v-i w(0)}{2 J+1}\right), \\
\mu^{-J} r^{-J},
\end{array}\right.
\end{aligned}
$$

and the regular solution at $r \rightarrow+\infty$ is defined as:

$$
\Phi=e^{-\mu r}\left(\begin{array}{c}
1 \\
\frac{-\mu}{i \lambda+m}
\end{array}\right) .
$$

In all those formulas, one has set $\mu=\sqrt{\lambda^{2}+m^{2}}$. Now we define the Jost function through:

$$
\begin{gathered}
\Phi(J, r)=F^{+}(J) f^{-}(r, J)-F^{-}(J) f^{+}(r, J), \\
F_{\mathrm{JOST}}(J)=F^{+}(J), \quad \operatorname{Re} J>0, \\
F_{\mathrm{JOST}}(J)=F^{-}(J), \quad \operatorname{Re} J<0 .
\end{gathered}
$$

They can be written in terms of the wronskian of two solutions $\Psi_{1}$ and $\Psi_{2}$ :

$$
W\left[\Psi_{1}\left(J_{1}\right) ; \Psi_{2}\left(J_{2}\right)\right]=-\Psi_{1}^{(1)} \Psi_{2}^{(2)}+\Psi_{1}^{(2)} \Psi_{2}^{(1)} .
$$

One clearly has:

$$
\begin{array}{ll}
F^{+}(J)=W\left(\Phi(J), f^{+}(J)\right), & \operatorname{Re} J>0, \\
F^{-}(J)=W\left(\Phi(J), f^{-}(J)\right), & \operatorname{Re} J<0,
\end{array}
$$

and conversely:

$$
\begin{aligned}
& f^{+}=F^{+} \Xi+G^{+} \Phi, \\
& f^{-}=F^{-} \Xi+G^{-} \Phi,
\end{aligned}
$$


where $\Xi$ is an exponentially increasing solution normalized by:

$$
\Xi_{r \rightarrow+\infty}^{=} e^{\mu r}\left(\begin{array}{c}
1 \\
+\mu \\
m-i \lambda
\end{array}\right), \quad W\left(\begin{array}{l}
W \\
\left.\Xi^{J}, \Phi^{J}\right)=1
\end{array}\right.
$$

All those identities follows from the wronskian property:

$$
\begin{aligned}
& \frac{d}{d r} W\left[\Psi_{1}\left(J_{1}\right), \Psi_{2}\left(J_{2}\right)\right] \\
& \quad=\frac{\left(J_{1}-J_{2}\right)}{r}\left[\Psi_{1}^{(1)}\left(J_{1}\right) \Psi_{2}^{(2)}\left(J_{2}\right)+\Psi_{1}^{(2)}\left(J_{2}\right) \Psi_{2}^{(1)}\left(J_{1}\right)\right],
\end{aligned}
$$

whose immediate consequence is:

$$
W\left(\Psi_{1}(J), \Psi_{2}(J)\right)=\text { const. }
$$

This property shall be extensively used to compute "scalar products" (3.6) (which shows again the interest of this definition!).

In the free case $(v=w=0)$, one can get exact solutions. Eliminating one of the two components in (3.1) and normalizing adequately leads to Bessel functions:

$$
\begin{gathered}
f_{0}^{+}(\varrho, J)=2^{J-1 / 2} \Gamma\left(J+\frac{1}{2}\right) \sqrt{\varrho}\left(\begin{array}{c}
I_{J-1 / 2}(\varrho) \\
\frac{m-i \lambda}{\mu} I_{J+1 / 2}(\varrho)
\end{array}\right), \\
f_{0}^{-}(\varrho, J)=2^{-J-1 / 2} \Gamma\left(\frac{1}{2}-J\right) \sqrt{\varrho}\left(\begin{array}{c}
\frac{m+i \lambda}{\mu} I_{1 / 2-J}(\varrho) \\
K_{-1 / 2-J}(\varrho)
\end{array}\right), \\
\Phi(\varrho, J)=+\sqrt{\frac{2 \varrho}{\pi}}\left(\begin{array}{c}
K_{J-1 / 2}(\varrho) \\
\frac{\mu}{m+i \lambda} K_{J+1 / 2}(\varrho)
\end{array}\right),
\end{gathered}
$$

where $\varrho=\left(\sqrt{\lambda^{2}+m^{2}}\right) r$ is dimensionless.

The Jost function reads:

$$
F_{0}^{ \pm}(J)=2^{-1 / 2 \pm J} \Gamma\left(\frac{1}{2} \pm J\right) .
$$

In the case when $v$ and $w$ are non-zero, we shall now define the spectrum of the operator $D_{I I}$. This spectrum consists of a discrete plus a continuum part.

1) The Jost solutions corresponding to the zeroes of $F^{+}$and $F^{-}$. These are the only regular solutions of Eq. (3.4).

2) The "continuum" solutions $\Phi(i \tau)$, for $\tau \in]-\infty,+\infty[$. As we shall see, this set of solutions forms a complete base of orthogonal functions. Using the "scalar product" (3.6) and the relation (3.15) together with the normalization conditions of the eigenfunctions, one gets, in particular:

- eigenfunctions associated to different eigenvalues $J_{K}$ are orthogonal, continuous and discrete eigenfunctions are orthogonal,

$$
\left\langle\Phi(i \tau), \Phi\left(i \tau^{\prime}\right)\right\rangle=\delta\left(\tau-\tau^{\prime}\right) F^{+}(i \tau) F^{-}(i \tau) .
$$


To prove completeness, we have to introduce the "Green function" associated to the operator $D_{I I}$. We define it through:

$$
D_{I I}(J) G\left(\varrho, \varrho^{\prime}, J\right)=\mathbb{1} \delta\left(\varrho-\varrho^{\prime}\right) .
$$

It is easy to show that the matrix operator $G$ defined by:

$$
\begin{aligned}
G\left(\varrho, \varrho^{\prime}, J\right)= & -\frac{i}{F(J)}\left(\left[f(\varrho, J) \otimes \Phi\left(\varrho^{\prime}, J\right)\right] \Theta\left(\varrho^{\prime}-\varrho\right)\right. \\
& \left.+\left[f\left(\varrho^{\prime}, J\right) \otimes \Phi(\varrho, J)\right] \Theta\left(\varrho-\varrho^{\prime}\right)\right) \sigma_{3}
\end{aligned}
$$

(where $J$ can be positive or negative, $f$ is respectively $f^{+}$or $f^{-}$, and $\theta$ is the Heaviside function) fulfills this equation together with the required boundary conditions $G\left(\varrho, \varrho^{\prime}, J\right) \rightarrow 0$ when $\left|\varrho-\varrho^{\prime}\right|$ becomes large.

We shall now compute in two ways the following integral:

$$
\lim _{R \rightarrow+\infty} \frac{1}{2 \pi i} \oint_{\Gamma} d J \int_{0}^{+\infty} \frac{d \varrho^{\prime}}{\varrho^{\prime}} G\left(\varrho, \varrho^{\prime}, J\right) \alpha\left(\varrho^{\prime}\right),
$$

where $\alpha(\varrho)$ is an arbitrary function,

$$
\Gamma=\Gamma^{+} \cup \Gamma^{-}
$$

is a contour which goes from $-i \infty+(-) \varepsilon$ to $+i \infty+(-) \varepsilon$ and closes to the left (right) with a circle of radius $R$. It is straightforward algebra to see that $J$-integration over the circle will generate a $\delta\left(\varrho-\varrho^{\prime}\right)$, and integrating over the two straight lines $]-i \infty \pm \varepsilon, i \infty \pm \varepsilon[$ generates an integral over the continuous scattering data. The global integral can be evaluated by Cauchy's theorem, and since the only poles in $G(J)$ correspond to $F\left(J_{K}\right)=0$, and therefore to eigenvalues of $D$, we are finally left with the following completeness relation:

$$
\begin{aligned}
& {\left[\sum_{K} c_{K} \Phi_{K}(\varrho) \otimes \Phi_{K}\left(\varrho^{\prime}\right)-\int_{-\infty}^{+\infty} \frac{\Phi(i \tau, \varrho) \otimes \Phi\left(i \tau, \varrho^{\prime}\right)}{F^{+}(i \tau) F^{-}(i \tau)} d \tau\right] \sigma_{1}} \\
& \quad=\varrho \delta\left(\varrho^{\prime}-\varrho\right) \cdot \mathbb{1},
\end{aligned}
$$

where $\Phi_{K} \equiv \Phi\left(J_{K}, \varrho\right) ; F\left(J_{K}\right)=0$, which shows that our definition of the spectrum of the operator $D_{I I}$ is consistent. Here,

$$
c_{K}^{ \pm}=\frac{1}{F^{\mp}\left(J_{K}\right) F^{\prime \pm}\left(J_{K}\right)} \quad\left(\operatorname{Re} J_{K} \gtrless 0\right) .
$$

Now that we have obtained all these preliminary relations, we shall define the scattering data of this problem, and we shall relate those S.D. with the Fredholm determinant $\ln \left(\frac{D_{I I}}{D_{I I}^{0}}\right)$. We define:

$-\left\{J_{K}\right\}_{K=1}^{N_{B}^{ \pm}}$which are the zeroes of the Jost function, and correspond therefore, as we have already seen, to regular solutions. We define:

$$
J_{K}^{+} \leftrightarrow \operatorname{Re}\left(J_{K}\right)>0 ; \quad J_{K}^{-} \leftrightarrow \operatorname{Re}\left(J_{K}\right)<0 .
$$

- $\left\{c_{K}\right\}_{K=1}^{N_{B}^{ \pm}}$, which are the normalization coefficients of the eigenfunctions $\Phi_{K}$ with respect to the "scalar product" (3.6). They read: 


$$
c_{K}=\frac{1}{\left(\int_{0}^{+\infty} \frac{2 d \varrho}{\varrho} \varphi_{K}^{(1)}(\varrho) \varphi_{K}^{(2)}(\varrho)\right)}=(\operatorname{cf} .(3.22)) \frac{-1}{F^{\mp}\left(J_{K}\right) F^{\prime \pm}\left(J_{K}\right)}
$$

[this follows from (3.6), (3.15)].

$$
D(\tau)=\frac{F^{+}(i \tau) F^{-}(i \tau)}{F_{0}^{+}(i \tau) F_{0}^{-}(i \tau)} \quad \text { (continuous contribution). }
$$

We shall prove later that this set of scattering data is sufficient to solve inverse scattering problems (up to the resolution of a Volterra-like integral equation). Several properties can be derived here.

If $v$ and $w$ are real functions of $r$, one can show that every eigenvalue $J_{K}$ must verify $J_{K}=-J_{K}^{*}$. This follows from computing:

$$
\begin{aligned}
& \int_{0}^{+\infty}\left[\varrho \varphi^{*(1)}\left(d_{\varrho} \varphi^{(2)}+(m+v+i w) \varphi^{(1)}\right)-\varrho \varphi^{*(2)}\left(d_{\varrho} \varphi^{(1)}+(m+v-i w) \varphi^{(2)}\right)\right] d \varrho \\
& =J \int_{0}^{+\infty} d \varrho\left[\varphi^{(1)} \varphi^{(1) *}+\varphi^{(2)} \varphi^{(2) *}\right] .
\end{aligned}
$$

Taking its complex conjugate gives $J=-J^{*}$. However, any eigenvalue $J_{K}$ has to satisfy $\operatorname{Re} J_{K} \neq 0$. Otherwise $\varphi_{K}^{(1)(2)} \sim \varrho^{J}$ is not regular when $\varrho \rightarrow 0$. Hence no discrete eigenvalue exists in this case (see [27]).

If the potential $w$ is zero, which corresponds to the Gross-Neveu effective action, a supplementary symmetry exists in the linear system. Namely, one can exchange the roles of $\varphi^{(1)}$ and $\varphi^{(2)}$, by changing $(J, \lambda)$ into $(-J,-\lambda)$ in the linear system. This leads to the following properties:

$$
\begin{gathered}
F_{(\lambda)}^{+}(J)=F_{(\lambda)}^{-}(-J), \\
F_{(\lambda)}\left(J_{K}\right)=0 \Leftrightarrow F_{(\lambda)}\left(-J_{K}\right)=0, \\
c_{K}\left(J_{K}\right)=c_{-K}\left(-J_{K}\right), \\
D_{(\lambda)}(\tau)=D_{(-\lambda)}(-\tau) .
\end{gathered}
$$

It is now possible to relate the scattering data to the Fredholm determinant $\ln \operatorname{det} \frac{D_{I I}}{D_{I I}^{0}}$. Using the identity that gives the Green function of $D_{I I},\left\langle\varrho\left|\frac{1}{D_{I I}}\right| \varrho^{\prime}\right\rangle$, we get an expression for the following quantity:

$$
\begin{aligned}
& \frac{d}{d J} \ln \operatorname{det}\left(\frac{D_{I I}(J)}{D_{I I}^{0}(J)}\right) \\
& \quad=\lim _{\varepsilon \rightarrow 0} \int_{\varepsilon}^{+\infty} i \sigma_{2} \frac{d \varrho}{\varrho} \operatorname{tr}\left[G(\varrho, \varrho, J)-G^{0}(\varrho, \varrho, J)\right],
\end{aligned}
$$

where $G^{0}$ is the free Green function that can be computed exactly from (3.16)-(3.18), and (3.20). Introducing the definition of $G$ together with the wronskian property (3.15), we can compute the integral in (3.23). Straightforward computation leads to:

$$
\frac{d}{d J} \ln \operatorname{det} \frac{D_{I I}(J)}{D_{I I}^{0}(J)}=\frac{d}{d J} \ln \frac{F(J)}{F_{0}(J)} .
$$


Since the limit of $\ln \frac{F(J)}{F_{0}(J)}$ and $\ln \operatorname{det} \frac{D_{I I}(J)}{D_{I I}^{0}(J)}$ when $|J| \rightarrow+\infty$ is clearly 0 (from the definition of $F$, and from expansion of $\left.\ln \operatorname{det} \frac{D_{I I}(J)}{D_{I I}^{0}(J)}\right)$, we end up with the very
important relation:

$$
\ln \operatorname{det} \frac{D_{I I}(J)}{D_{I I}^{0}(J)}=\ln \operatorname{det} \frac{F(J)}{F_{0}(J)} .
$$

Again, as in the previous case of the Schrödinger equation, this relation between the determinant of a differential operator and an associated "Jost function" is a particular case of a general theorem [28]. Using now a standard dispersion relation for $F(J)\left(F^{+}\right.$being analytic for $\operatorname{Re} J>0$ and $F^{-}$being analytic for $\operatorname{Re} J<0)$ :

$$
\begin{aligned}
\frac{F^{ \pm}(J)}{F_{0}^{ \pm}(J)}= & \prod_{K=1}^{N_{B}^{ \pm}}\left(\frac{J-J_{K}^{ \pm}}{J+J_{K}^{ \pm}}\right) \exp \mp \frac{1}{2 \pi} \int_{-\infty}^{+\infty} \frac{d \tau^{\prime}}{i \tau^{\prime}-J} \\
& \cdot\left[\ln D\left(\tau^{\prime}\right)+\sum_{K=1}^{N_{B}} \ln \left(\frac{i \tau^{\prime}+J_{K}}{i \tau^{\prime}-J_{K}}\right)\right],
\end{aligned}
$$

we obtain therefore an expression of the functional determinant $\operatorname{det} \frac{D_{I I}(J)}{D_{I I}^{0}(J)}$ as a local function of the associated scattering data. Notice that such a relation has already been obtained for the Dirac operator with energy as spectral parameter, and closely looks like (3.26) [27]. From now on, we shall always consider $\lambda=0$. This is justified by the fact that all the results which we shall now derive are used in the article after dimensional regularization has been achieved, which amounts to setting $\lambda=0$. We therefore need not introduce this supplementary variable, and we shall omit it for the sake of simplicity. The problem now is to try to reexpress the local terms in $w$ which appear in the effective action, in a local form as functions of the scattering data. We shall compute the corresponding "trace identity."

Relations between integral of potential and scattering data can generally be obtained by expanding both sides of Eq. (3.25) in powers of $J^{-1}$ and identifying the integrals of the potentials that appear on the left-hand side with the functions of S.D. that appear on the right-hand side, order by order in $1 / J$. It is here possible (as in the Schrödinger case) to proceed more quickly. We set:

$$
\begin{aligned}
& f_{(1)}(r)(\text { Jost solution })=f_{(1)}^{0} e^{\psi(r)} \Rightarrow \psi(0)=0, \\
& \begin{array}{l}
\text { (regular at } r \rightarrow 0) \\
(\operatorname{Re} J>0)
\end{array}(+\infty)=\ln \frac{F(J)}{F_{0}(J)} .
\end{aligned}
$$

Inserting this into the system (3.4), we get the following Ricatti equation

$$
\psi^{\prime \prime}+\psi^{\prime 2}+2 \alpha^{\prime} \psi^{\prime}-2 m v-v^{2}-w^{2}+\left(\frac{v^{\prime}-i w^{\prime}}{m+v-i w}\right)\left(\psi^{\prime}+\alpha^{\prime}-J / \varrho\right)=0
$$

where we have set

$$
\begin{gathered}
\alpha^{\prime} \equiv \frac{d}{d r} \ln f_{(1)}^{0}(r) \\
f_{(1)}^{0}(r)=\sqrt{\varrho} \cdot I_{J-1 / 2}(\varrho) \cdot(\text { constant }) .
\end{gathered}
$$


We know that

$$
\frac{F(J)}{F_{0}(J)}=\psi(+\infty)=\int_{0}^{+\infty} \psi^{\prime}(r) d r
$$

We set:

$$
\alpha^{\prime}(r)=\frac{J}{r}+\sum_{n \geqq 1} \frac{Q_{n}(r)}{J^{n}} .
$$

$\left[Q_{n}(r)\right.$ can be obtained exactly since $f_{1}^{0}$ is known analytically. $]$ One gets:

$$
\begin{aligned}
& Q_{1}(r)=m^{2} r / 2, \\
& Q_{2}(r)=-m^{2} r / 2 .
\end{aligned}
$$

We set:

$$
\begin{aligned}
& \psi^{\prime}(r)=\sum_{n \geqq 1} \frac{h_{n}(r)}{J^{n}}, \\
& \frac{F(J)}{F_{0}(J)}=\sum_{n \geqq 1} \frac{c_{n}}{J^{n}} .
\end{aligned}
$$

The $c_{n}$ can be obtained straightforwardly by expanding the dispersion relation (3.26) in powers of $1 / J$; their generic form is

$$
\sum_{K=1}^{N_{B}} p_{n}\left(J_{K}\right)-\int_{-\infty}^{+\infty} p_{n}^{\prime}(i \tau) \ln D(\tau) d \tau
$$

where $p_{n}$ is an $n$-degree polynomial.

Solving now the Ricatti equation (3.28), order by order in $1 / J$, and using relations (3.29), one obtains the following solutions:

$$
\begin{gathered}
h_{1}(r)=\frac{1}{2} r\left(v^{2}+2 m v+w^{2}\right), \\
\frac{2 h_{n}}{r}+h_{n-1}^{\prime}+\sum_{\substack{p>0 \\
p^{\prime}>0 \\
p^{\prime}=n-1}} h_{p} h_{p^{\prime}}+\sum_{\substack{p>0 \\
p^{\prime}>-1 \\
p+p^{\prime}=n-1}} Q_{p^{\prime}} h_{p} \\
+\left(\frac{v^{\prime}-i w^{\prime}}{m+v-i w}\right)\left(h_{n-1}+Q_{n-1}\right)=0,
\end{gathered}
$$

and $\left[c_{n}=\int_{0}^{+\infty} h_{n}(r) d r\right]$ are the trace identities. We shall give explicitly the first trace identity, since it is the only one that is used in this article.

$$
\frac{1}{2} \int_{0}^{+\infty} r\left(v^{2}+2 m v+w^{2}\right) d r=\sum_{K=1}^{N_{B}}\left(-\operatorname{sgn} \operatorname{Re} J_{K}\right) J_{K}+\int_{0}^{+\infty} \frac{\ln D(\tau)}{2 \pi} d \tau .
$$

Forthcoming papers will use higher order trace identities that will be given then.

There remains now to write the inverse scattering formula, and to obtain explicit expressions for the functional derivatives of $v$ with respect to the S.D., and reciprocally. We shall here show that the scattering data defined above are a 
complete set in that they fully determine the potentials $v$ and $w$, and there exists a biunivocal relation between the two "sets of fields." As usual, we define a (matrix) Kernel $K$ which connects free to interacting solutions ( $\Phi$-like solutions!)

$$
\left\{\begin{array}{l}
\Phi(J, \varrho)=\int_{\varrho}^{+\infty} K\left(\varrho, \varrho^{\prime}\right) \Phi_{0}\left(J, \varrho^{\prime}\right) d \varrho^{\prime}+\Phi_{0}(J, \varrho), \\
K\left(\varrho, \varrho^{\prime}\right)=0, \text { when } \varrho>\varrho^{\prime} .
\end{array}\right.
$$

From this definition of $K$, and using the closure relation (3.21) one can show, after a long but straightforward computation, that $K$ is the solution of a Gel'fandLevitan-like matrix equation

$$
\left\{\begin{array}{l}
K\left(\varrho, \varrho^{\prime}\right)+\Omega\left(\varrho, \varrho^{\prime}\right)+\int_{0}^{+\infty} \Omega\left(\varrho, \varrho^{\prime \prime}\right) K\left(\varrho^{\prime \prime}, \varrho^{\prime}\right) d \varrho^{\prime \prime}=0 \quad\left(\varrho<\varrho^{\prime}\right), \\
K\left(\varrho, \varrho^{\prime}\right)=0 \quad\left(\varrho>\varrho^{\prime}\right)
\end{array}\right.
$$

where $\Omega$ is a function of the scattering data:

$$
\begin{aligned}
\Omega\left(\varrho, \varrho^{\prime}\right)= & -\int_{-\infty}^{+\infty} \frac{d \tau}{\pi}\left(\Phi_{0}(i \tau, \varrho) \otimes \Phi_{0}\left(i \tau, \varrho^{\prime}\right)\right)\left(\frac{1}{D(\tau)}-1\right)(\sinh \pi \tau) \\
& +\sum_{K=1}^{N_{B}} c_{K} \Phi_{0}\left(J_{K}, \varrho\right) \otimes \Phi_{0}\left(J_{K}, \varrho^{\prime}\right) .
\end{aligned}
$$

This equation has a unique solution $K\left(\varrho, \varrho^{\prime}\right)$ if the scattering data $\left\{D(\tau), c_{K}, J_{K}\right\}$ verify properties analogous to the properties required in the case of the Schrödinger equation with an angular momentum variable. This shows in fact that $\left\{\lambda_{K}, c_{K}, D(\tau)\right\}$ is a complete set of S.D., and justifies our replacing $\{v, w\}$ by $\{$ S.D. $\}$ in our problem. One can obtain $v$ and $w$ from $K$ : introducing the operators $D_{I I}$ and $D_{I I}^{0}$, one gets:

$$
V *(\mathbb{1}+K)=\left[D_{I I}^{0}, K\right], \text { where } D_{I I}=D_{I I}^{0}+V .
$$

Simple algebraic manipulations lead to:

$$
\begin{gathered}
v(r)=-\frac{1}{r} \operatorname{Tr}\left(\sigma_{1} K(\varrho, \varrho)\right), \\
i w(r)=-\frac{i}{r} \operatorname{Tr}\left(\sigma_{2} K(\varrho, \varrho)\right), \\
-\varrho^{\prime} \cdot \frac{d}{d \varrho} K\left(\varrho, \varrho^{\prime}\right) \cdot \sigma_{3}+\varrho \cdot \sigma_{3} \cdot \frac{d}{d \varrho} K\left(\varrho, \varrho^{\prime}\right)-i r^{\prime} K\left(\varrho, \varrho^{\prime}\right) \sigma_{2} m \\
+i r \sigma_{2} K\left(\varrho, \varrho^{\prime}\right) m=r V K(\varrho, \varrho) .
\end{gathered}
$$

To complete our study, we need to compute derivatives of $v$ and $w$ with respect to the S.D. (which amounts to obtaining a differential expression for the G.L.M. equation). Using (3.35) and (3.32), (3.33), one gets $(m \equiv 1)$ :

$$
\begin{aligned}
\delta v(\varrho)= & \frac{1}{\varrho} \operatorname{Tr}\left(\Phi_{K} \otimes \Phi_{K}\right) \delta c_{K}+\frac{2}{\varrho} \operatorname{Tr}\left(\frac{d \Phi}{d J_{K}} \otimes \Phi_{K}\right) c_{K} \delta J_{K}-\frac{1}{\varrho} \\
& \cdot \int_{-\infty}^{+\infty} \frac{\sinh \pi \tau}{(D(\tau))^{2}} \delta D(\tau) \operatorname{Tr}(\Phi(i \tau) \otimes \Phi(i \tau)) d \tau,
\end{aligned}
$$


where the $\Phi$ are, of course, interacting $\Phi$-like solutions (i.e. exponentially decreasing).

The derivative, $i \delta w(\varrho)$, follows from replacing $\operatorname{Tr}(\Phi \otimes \Phi)$ by $\operatorname{Tr}\left[\Phi^{+} \sigma_{3} \Phi\right]$ in (3.36) [which we shall also note $\operatorname{Tr}^{\ominus}(\Phi \otimes \Phi)$ ]. We can invert (3.36) by using closure relations, or by direct perturbation theory. One gets for instance:

$$
\frac{\delta J_{K}}{\delta v(\varrho)}=c_{K} \Phi_{K}^{+} \sigma_{3} \Phi_{K} \equiv c_{K} \operatorname{tr}^{\ominus}\left(\Phi_{K} \otimes \Phi_{K}\right)
$$

and

$$
\frac{\delta J_{K}}{\delta w(\varrho)}=i c_{K} \Phi_{K}^{+} \Phi_{K},
$$

which shall be the most useful inverse functional derivatives when studying $S_{\text {eff }}$. (Here $\operatorname{tr}^{\ominus}$ on a $2 \times 2$ matrix $M$ means taking the trace of $\sigma_{3} \cdot M$.)

\section{The Effective Action and S.D. Analysis}

Let us now come back to our main problem, which is to recast $S_{\text {eff }}$ in (2.32), as far as possible, as a local functional of the scattering data previously introduced. We know that the Fredholm determinant coincides with the Jost function, and can henceforth be expressed in terms of the S.D.:

$$
\begin{aligned}
\operatorname{Det} \frac{D_{I I}(J, \lambda)}{D_{I I}^{0}(J, \lambda)}= & \frac{F^{+}(J)}{F_{0}^{+}(J)}=\prod_{K^{+}} \frac{J-J_{K}^{+}}{J+J_{K}^{+}} \exp -\frac{1}{2 \pi} \int_{-\infty}^{+\infty} \frac{d \tau}{i \tau-J} \\
& \cdot\left(\ln D(\tau)+\sum_{K} \ln \left(\frac{i \tau+J_{K}}{i \tau-J_{K}}\right)\right)(\operatorname{Re}(J)>0), \\
(\operatorname{Re}(J)<0)= & \frac{F^{-}(J)}{F_{0}^{-}(J)}=\prod_{K} \frac{J-J_{K}^{-}}{J+J_{K}^{-}} \exp \frac{1}{2 \pi} \int_{-\infty}^{+\infty} \frac{d \tau}{i \tau-J} \\
& \cdot\left(\ln D(\tau)+\sum_{K} \ln \left(\frac{i \tau+J_{K}}{i \tau-J_{K}}\right)\right),
\end{aligned}
$$

where $D(\tau), J_{K}^{+}, J_{K}^{-}$are the scattering data previously introduced. Moreover, we also recognize in (2.38) the first trace identity (TR 1 ), derived in Sect. III. The logarithmic term in (2.38) can also be expressed in closed form as a local functional of the scattering data with the help of a non-standard trace identity [see (1.15)]. ["Non-standard" means here that, as it was the case for Schrödinger equation with angular momentum, we had to derive it by hand, unlike (TR1) or (3.30).] Using now (TR1):

$$
\frac{1}{2} \int_{\sigma}^{+\infty} r d r\left(v^{2}+2 m v+w^{2}\right)=-\sum_{K}\left(\operatorname{sgn} \operatorname{Re} J_{K}\right) J_{K}+\frac{1}{2 \pi} \int_{-\infty}^{+\infty} \ln D(\tau) d \tau,
$$

and the non-standard trace identity (1.15), together with the functional identity [29]:

$$
\sum_{\ell=0}^{+\infty}\left[\ln \left(\frac{\ell+\frac{1}{2}-z}{\ell+\frac{1}{2}+z}\right)-\frac{2 z}{\ell+\frac{1}{2}}\right]=\log \frac{\Gamma\left(\frac{1}{2}+z\right)}{\Gamma\left(\frac{1}{2}-z\right)}+(2 z)(\gamma+2 \ln 2)
$$


enables one to obtain the effective action as a local functional of the scattering data (except for a single term, which we shall discuss below). This fully justifies the use of IST. to simplify the study of this effective action. $S_{\text {eff }}$ reads:

$$
\begin{aligned}
S_{\mathrm{eff}}= & -\frac{1}{4 \pi^{2}} f_{-\infty}^{+\infty} \frac{d \tau d \tau^{\prime}}{\left(\tau-\tau^{\prime}\right)^{2}} \ln D(\tau) \ln D\left(\tau^{\prime}\right) \\
& -\frac{1}{\pi} \int_{-\infty}^{+\infty}\left(\sum_{K} \frac{\operatorname{sgn} \operatorname{Re} J_{K}}{J_{K}-i \tau}\right) \ln D(\tau) d \tau+\sum_{K} \ln \frac{\cos \pi J_{K}}{2 \pi} \\
& -2 \sum_{K \neq K^{\prime}} \operatorname{sgn} \operatorname{Re} J_{K} \operatorname{sgn} \operatorname{Re} J_{K}^{\prime} \ln \left(J_{K}-J_{K}^{\prime}\right) \\
& -\sum_{K} \ln c_{K}+\frac{1}{2 g_{R}} \int_{0}^{+\infty} r d r w^{2}(r) .
\end{aligned}
$$

Several remarks must now be made concerning this effective action.

1) Terms linear in the scattering data $\ln D(\tau)$ and $J_{K}$ were present in (4.2), (4.3), and [12], but they cancel in $S_{\text {eff }}$ (4.4). This was to be expected since such terms are associated with the only trace identity containing a linear part in $v$. Since $S_{\text {eff }}$ is quadratic in $v$ (for $v$ small), no linear term in the S.D. can appear in it. The same feature appeared for instance in the $N L \sigma$-model in 2 dimensions $[5,6,9]$.

2) The $\Gamma$ functions [12] and (4.3) combine in (4.4) to form trigonometric functions. This phenomenon was also to be expected, since a $\ln \Gamma\left(J_{K}\right) \underset{J_{K} \rightarrow+\infty}{\sim} J_{K} \ln J_{K}$ behaviour is not natural for such an effective action. Again, this was also observed before for bosonic theories $[2,5]$.

3) It does not seem possible to rewrite the last term in $S_{\text {eff }}(4.4)$ in closed form as a function of the scattering data. It is not a standard trace identity, neither does it appear as directly derivable by computation of functional derivatives with respect to the S.D., as in the Appendix. On the other hand, this term is absent in two cases: $w \equiv 0$, which corresponds to the Gross-Neveu model, and $1 / g_{R}=0$, which corresponds to the Chiral Gross-Neveu model. Those two theories are known to be integrable models at the quantum level [12-16]. We find here a feature that already appeared in bosonic models, such as the anharmonic oscillator, and the $N L \sigma$-model in two dimensions: integrability of the minkowskian model, and separation of the euclidean effective action expressed in terms of the S.D. seem to go together.

We can now investigate the instantons of the effective action, which will give us indications about the behaviour of the $1 / N$ expansion. We shall not here make numerical computations, and restrict ourselves to an analytic investigation of the limiting cases $g_{R} \rightarrow 0^{ \pm}$and $g_{R} \rightarrow \pm \infty$. Indeed, numerical computations would be longer than in the case of bosonic models, owing to the presence of two potentials. Moreover, the effective action exhibits a property that will make such an investigation more difficult: in the bosonic case [5], the configuration space of fields was separated into homotopy classes labelled by the number of bound states, and separated by infinite action barriers. This is no more the case in this twopotential problem, since the $J_{K}$ can be arbitrary complex numbers. Hence $S_{\text {eff }}$ does not exhibit infinite barriers between sectors with different $N_{B}$, but rather infinite action lines each time some $J_{K}$ equals $1 / 2$ plus an integer. One could think when 
examining (4.4) that $J_{K}=0$ is also a singularity. However, it is clear that each term of the effective action, taken individually, is not divergent when $J_{K} \rightarrow 0$. Any numerical analysis will have to extend over all the functional space.

1. Integrable Models: Gross-Neveu and CGN Model. The integrable character of these models reflects itself, as we have seen, in the closed form of $S_{\text {eff }}$ expressed in the S.D. It is therefore possible to analyze the saddle-points of $S_{\text {eff }}$, without any approximation, by extremizing independently with respect to each S.D. Extremizing with respect to $D(\tau)$ gives:

$$
0=\frac{\delta S}{\delta D(\tau)}=\frac{2}{\pi D(\tau)} \frac{d}{d \tau} \delta(\tau)
$$

where

$$
\delta(\tau) \equiv \ln \left(\frac{F^{+}(i \tau)}{F_{0}^{+}(i \tau)} \frac{F_{0}^{-}(i \tau)}{F^{-}(i \tau)}\right)
$$

This follows from the fact that linear terms in the $\ln \operatorname{det}\left(\frac{D_{I I}}{D_{I I}^{0}}\right)$, $\int_{0}^{+\infty} r \ln m r\left(v^{2}+2 m v+w^{2}\right) d r$, and $\int_{0}^{+\infty} r\left(v^{2}+2 m v+w^{2}\right) d r$, cancel, while there only subsist in $S_{\text {eff }}$ terms in $\ln D(\tau)$ coming from the non-linear part in the non-standard trace identity. Equation (4.6) follows immediately. Let us introduce now the dispersion relation (not derived in Sect. III) that expresses $F(J)$ as a functional of $\left\{J_{K}, \delta(\tau)\right\}$ :

$$
\begin{aligned}
\frac{F^{ \pm}(J)}{F_{0}^{ \pm}(J)}= & \prod_{K^{ \pm}} \frac{J-J_{K}^{ \pm}}{J+J_{K}^{ \pm}} \exp -\frac{1}{2 \pi} \int_{-\infty}^{+\infty} \frac{d \tau^{\prime}}{i \tau^{\prime}-J} \\
& \cdot\left(i \delta(\tau)+\sum_{K^{+}} \ln \left(\frac{i \tau+J_{K}}{i \tau-J_{K}}\right)-\sum_{K^{-}} \ln \left(\frac{i \tau+J_{K}}{i \tau-J_{K}}\right)\right) .
\end{aligned}
$$

Assuming that $\delta(+\infty)=0$ as a normalization condition on the Jost function, we find from (4.6) that $\delta(\tau) \equiv 0$. We finally get, after integrating in (4.8):

$$
D(\tau)=\prod_{K}\left(\frac{\tau+i J_{K}}{\tau}\right)^{2}
$$

Extremizing with respect to the discrete eigenvalue $J_{K}$ leads to:

$$
\begin{aligned}
0= & \frac{\delta S}{\delta J_{K}}=\pi \tan \pi J_{K}-2 \sum_{K \neq K^{\prime}} \frac{\operatorname{sgn}\left(\operatorname{Re} J_{K}\right) \operatorname{sgn}\left(\operatorname{Re} J_{K}^{\prime}\right)}{\left(J_{K}-J_{K}^{\prime}\right)} \\
& +\frac{1}{\pi} \int_{-\infty}^{+\infty} \operatorname{sgn}\left(\operatorname{Re} J_{K}\right) \frac{\ln D(\tau)}{\left(J_{K}-i \tau\right)^{2}} d \tau .
\end{aligned}
$$

Inserting (4.9) into (4.10) leads to:

$$
\pi \tan \pi J_{K}+\sum_{L \neq K} \frac{\operatorname{sgn} \operatorname{Re} J_{K} \operatorname{sgn} \operatorname{Re} J_{L}}{J_{K}-J_{L}}+\sum_{L} \frac{4}{J_{K}-J_{L}}=0 .
$$

Finally, extremizing with respect to $c_{K}$ gives:

$$
0=\frac{\delta S}{\delta c_{K}}=\frac{1}{c_{K}} \text {. }
$$


This cannot lead to any finite action maximum or minimum. Moreover, if a solution to the gap equation exists with a non-zero eigenvalue $J_{K}$, it will lead to a singular field $v, w$ near $r=0$, since $\ln D(\tau)$ given by (4.9) vanishes too slowly when $\tau \rightarrow+\infty: \ln D(\tau) \sim \frac{\left(\sum_{K} J_{K}\right)}{\tau}$.

Finally, Levinson's theorem fails if $N_{B} \neq 0$, since it implies that $\delta(0)=\tau N_{B}$, while $\delta(\tau)=0$ from (4.6). However, it is interesting to investigate those "singular instantons," because the general gap equation looks exactly like these equations, apart from a term $1 / g_{R} \int_{0}^{+\infty} w(r) \cdot \frac{\delta w}{\delta S D} \cdot r d r$. Hence we expect that when $\left|g_{K}\right| \rightarrow+\infty$, the instantons (if any) of the general fermionic models will tend towards these "limit instantons" of the CGN model. Note that we do not know a priori whether inverse scattering on the solution-S.D. will generate limit-GN $(w \equiv 0)$ or CGN $(w \neq 0)$ like potentials. We know that "Gross-Neveu" instantons have the following features:

$$
\begin{aligned}
F\left(J_{K}\right)=0 \Leftrightarrow & F\left(-J_{K}\right)=0, \quad J_{-K} \equiv-J_{K}, \\
& D(\tau)=D(-\tau), \\
& c_{K}=c_{-K},
\end{aligned}
$$

following from the property $F(J)=F(-J)$. Hence, depending on whether solutionS.D. verify or not these properties we shall obtain "GN" or "CGN" instantons.

Anyhow we can already, before investigating gap equations, draw some exact conclusions for the two integrable models. Since no instanton exists with a finite action, we are bound to conclude that the $1 / N$ expansion is probably more convergent than the usual $K ! / a^{K}$ behaviour. This conclusion is confirmed by the exact results on the $S$-matrix for these models $[13,14]$ : the $S$-matrix exhibits in fact a convergent $1 / N$ expansion. It is possible (as it is also possible in the case of the $N L-\sigma$ model, $[30,2,15])$ that this property should extend to off-shell quantities, but we have no indication on this question (see below for supplementary discussions).

Let us now discuss the gap equation (4.11). The simplest possible set of solutions compatible with the properties of S.D. is a couple of real solutions $J_{K},-J_{K}$. Equation (4.11) then reads:

$$
\pi \tan \pi J_{K}-\frac{1}{J_{K}}=0,
$$

which has an infinite set of solutions that can be numerically computed. Note that since $J_{K}$ and $-J_{K}$ are real, $D(\tau)$ will read:

$$
D(\tau)=\left(\frac{\tau+i J_{K}}{\tau}\right)^{2}\left(\frac{\tau-i J_{K}}{\tau}\right)^{2}=D(-\tau) .
$$

These relations lead us to conjecture that the limit solutions $v$ and $w$ are such that $|v| \gg|w|$ so that the S.D. become those of a "Gross-Neveu" set of potentials (see Sect. III). Of course, we have no well-defined $c_{K}$ and we do not know whether $c_{K}=c_{-K}$ when $g_{R} \rightarrow-\infty$ (see below). 
2. Intermediate Models. $1 / g_{R} \neq 0 ; w \neq 0 ; g_{R}<0$ (no tachyons). The limit $g_{R} \rightarrow-\infty$ has already been partially discussed: In fact, it amounts to adding a $1 / g_{R} \int_{0}^{+\infty} w(r) \cdot \frac{\delta w}{\delta \text { S.D. }} \cdot r d r$ to all gap equations, with $\frac{\delta w}{\delta \text { S.D. }} \equiv$ functional derivative of $w$ with respect to the corresponding scattering data. Assuming that the limit potential $w$ is not too singular (it is the strongest possible assumption since we suspect, from our previous discussion, that at least one of the two limiting potentials $v$ and $w$ is singular) leads us to the limit S.D.: $D(\tau)=\prod_{K}\left(\frac{\tau-i J_{K}}{\tau}\right)^{2}$ and
$\left\{J_{K}\right\}$ solutions of (4.11).

The equation $\frac{\delta S}{\delta c_{K}}=0$ leads to:

$$
\frac{1}{c_{K}}+\frac{1}{g_{R}} \int_{0}^{+\infty} r w(r) \frac{\delta w}{\delta c_{K}} d r=0 .
$$

This means that if $w$ is not too singular, $c_{K}$ will behave like $g_{R}$ or more generally [as it happened in the limit $g_{R} \rightarrow-\infty$ of $\left(\Phi^{2}\right)_{I I}^{2}$-model $] c_{K} \sim\left|g_{R}\right|^{\alpha}, \alpha>0$. Since this term dominates the effective action [neither $D(\tau)$ nor $J_{K}$-dependent term can create a divergent behaviour in $S_{\text {eff }}$ when $\left.\left|g_{R}\right| \rightarrow+\infty\right]$ we expect that $S_{c} \sim-\alpha \ln \left|g_{R}\right|+\mathcal{O}(1)$. Let us now recall the structure of the "large-order integral" first derived by Lipatov [10].

$$
\mathscr{Z}(K)=\oint d N \iint \mathscr{D} v \mathscr{D} w e^{N S_{\text {eff }}(v, w)+K \ln N} .
$$

The double saddle-point equation reads:

$$
\frac{\delta S}{\delta v}=0 ; \quad \frac{\delta S}{\delta w}=0 ; \quad S_{c}(v, w)+\frac{K}{N_{c}}=0 \Rightarrow N_{c}=\frac{-K}{S_{c}} .
$$

Hence

$$
\mathscr{Z}(K) \sim e^{-K} \frac{K^{K}}{\left(-S_{c}\right)^{K}} .
$$

Owing to the fact that the saddle-points are a priori complex, $\mathscr{Z}(K)$ will probably exhibit a phase fluctuation due to $\left(-S_{c}\right)^{K}$. Hence the $1 / N$ expansion is Borelsummable in the neighborhood of $g_{R} \rightarrow-\infty$ [10]. This is correct, unless a real negative action instanton appears. Our present analysis cannot solve this problem: one should go further in the study of $S_{\text {eff }}$ and probably investigate it numerically to have an indication. Anyhow it clearly appears here that GrossNeveu and Chiral Gross-Neveu models have a special role amongst the other selfcoupled fermionic theories; their $1 / N$ expansion has much less divergent behaviour than in the other models, and could even be an entire series. This result can be compared with a recent exact result concerning perturbative $(g)$ expansion of the massive Gross-Neveu model $\mathscr{L}=\bar{\Psi}(\phi+m) \Psi+g(\bar{\Psi} \Psi)^{2}$ : perturbative expansion in $g$ is much more convergent than any and could even be summable [32].

We shall now briefly investigate the limit $g_{R} \rightarrow 0^{-}$of the instanton. The extremum with respect to $c_{K}$ reads:

$$
\frac{\delta S}{\delta c_{K}}=0=\frac{1}{c_{K}}+\frac{1}{g_{R}} \int_{0}^{+\infty} w(r) \operatorname{tr}^{\ominus}\left(\Phi_{K}(r) \otimes \Phi_{K}(r)\right) d r .
$$


Assuming that neither $w(r)$ nor $\Phi_{K}(r)$ are singular, we obtain that all normalization constants $c_{K}$ go to zero like $g_{R}$. Since $c_{K}\left(\operatorname{Re} J_{K}>0\right)=-1 / F^{-}\left(J_{K}\right) F^{\prime+}\left(J_{K}\right)$ (and $+\leftrightarrow-$ when $\left.\operatorname{Re} J_{K}<0\right)$, this means that $F^{-}\left(J_{K}\right)$ must have a singular behaviour. Note that $F^{-}$need not be analytic for $\operatorname{Re} J_{K}>0$, so that this property is consistent with the normal behaviour of Jost functions. The same argument holds for $\operatorname{Re} J_{K}<0$. Let us now study the complete gap equation:

$$
\begin{aligned}
\frac{\delta S}{\delta w(r)}=0 & \Rightarrow \frac{r w(r)}{g_{R}}+i \sum_{K}\left(\pi c_{K} \operatorname{Tr}\left(\Phi_{K} \otimes \Phi_{K}\right) \tan \pi J_{K}\right) \\
& + \text { continuum contributions }=0 .
\end{aligned}
$$

Unless $w(r)$ goes to zero when $g_{R}$ goes to zero $(-)$ [meaning that the theory has a Gross-Neveu-like limit ( $w \equiv 0)]$, we have to cancel in (4.18) the singular behaviour $1 / g_{R}$ by another singular behaviour. Such a cancellation is clearly realized if one of the eigenvalues (and, in fact, then two of the eigenvalues, as we shall see later) approaches a half-plus-integer value. Owing to the additivity of such singularities $\left(S_{\text {eff }} \sim \sum_{K} \ln \cos \pi J_{K}\right)$, and since the most important contributions to $1 / N$ large order behaviour come from the lowest-action instanton, we shall restrict ourselves to the smallest possible number of eigenvalues. The simplest possible case is then $J_{K}^{+}=\frac{1}{2}+\mathcal{O}\left(g^{2}\right)\left[\right.$ since $\left.c_{K} \sim \mathcal{O}(g)\right]$.

The second gap equation can be studied in a similar way:

$$
\begin{aligned}
\frac{\delta S}{\delta v(r)}= & 0=\sum_{K} \pi \cdot c_{K} \cdot \operatorname{tr}^{\ominus}\left(\Phi_{K} \otimes \Phi_{K}\right) \cdot \tan \pi J_{K} \\
& + \text { continuum contributions } .
\end{aligned}
$$

[See note following (3.31) for explanation of the " $\mathrm{tr}^{\ominus}{ }_{\text {-symbol. }}$. We therefore need at least $t w o$ critical eigenvalues; otherwise we shall get $0=$ singularity. If we have two eigenvalues $-\frac{1}{2}-\mathcal{O}\left(g^{2}\right)$ and $\frac{1}{2}+\mathcal{O}\left(g^{2}\right)$ when $g_{R} \rightarrow 0^{-}$, then Eq. (4.19) implies, to leading order in $(1 / g)$, that

$$
c_{K}^{+} \operatorname{tr}^{\ominus}\left(\Phi_{K}^{+} \otimes \Phi_{K}^{+}\right)=\alpha c_{K}^{-} \operatorname{tr}^{\ominus}\left(\Phi_{K}^{-} \otimes \Phi_{K}^{-}\right) \quad(\alpha \in \mathbb{C}) .
$$

It is difficult to go any further in (4.19) since we would have to study the (assumedly finite) continuum contributions. However, it is possible to draw conclusions on the saddle-points. Cancellation of singular parts in (4.17) and (4.18) implies, if we assume that neither $w(r)$ nor $\Phi_{K}$ are singular:

$$
\begin{gathered}
c_{K}^{+}=\alpha^{+} g_{R} ; \quad c_{K}^{-}=\alpha^{-} g_{R} ; \\
\cos \pi J_{K}^{+}=\beta^{+} \cdot g_{R}^{2} \cdot \pi ; \quad \cos \pi J_{K}^{-}=\beta^{-} \cdot g_{R}^{2} \cdot \pi ; \\
\sin \pi J_{K}^{+}=1 ; \quad \sin \pi J_{K}^{-}=-1 ; \\
J_{K}^{ \pm}(\text {simplest eigenvalues })= \pm \frac{1}{2} \pm \beta^{ \pm} g_{R}^{2}, \\
r w(r)=i\left[\frac{\alpha^{+}}{\beta^{+}} \operatorname{tr}\left(\Phi_{K}^{+} \otimes \Phi_{K}^{+}\right)-\frac{\alpha^{-}}{\beta^{-}} \operatorname{tr}\left(\Phi_{K}^{-} \otimes \Phi_{K}^{-}\right)\right], \\
\frac{\alpha^{+}}{\beta^{+}} \operatorname{tr}^{\ominus}\left(\Phi_{K}^{+} \otimes \Phi_{K}^{+}\right)=\frac{\alpha^{-}}{\beta^{-}} \operatorname{tr}^{\ominus}\left(\Phi_{K}^{-} \otimes \Phi_{K}^{-}\right) .
\end{gathered}
$$


Inserting (4.2) in the diffusion problem (2.12) leads to compatibility equations for $\Phi_{K}^{ \pm}$:

$$
\begin{aligned}
& \left\{\sigma_{3} d_{\varrho}-i \sigma_{2}(m+v)-i\left[\frac{\alpha^{+}}{\beta^{+}} \operatorname{tr}\left(\Phi_{K}^{+} \otimes \Phi_{K}^{+}\right)-\frac{\alpha^{-}}{\beta^{-}} \operatorname{tr}\left(\Phi_{K}^{-} \otimes \Phi_{K}^{-}\right)\right] \sigma_{1}\right\} \Phi_{K}^{+} \\
& \quad=-\Phi_{K}^{+} / 2 \varrho, \\
& \left\{\sigma_{3} d_{\varrho}-i \sigma_{2}(m+v)-i\left[\frac{\alpha^{+}}{\beta^{+}} \operatorname{tr}\left(\Phi_{K}^{+} \otimes \Phi_{K}^{+}\right)-\frac{\alpha^{-}}{\beta^{-}} \operatorname{tr}\left(\Phi_{K}^{-} \otimes \Phi_{K}^{-}\right)\right] \sigma_{1}\right\} \Phi_{K}^{-} \\
& =\Phi_{K}^{-} / 2 \varrho .
\end{aligned}
$$

It is possible to solve (4.25) and (4.24) numerically. However, it will not give us more indications about these instantons. In fact, Eq. (4.25) looks like a "field equation" for the Lagrangian $\mathscr{L}=\bar{\Psi} \not \Psi \Psi+g_{1}(\bar{\Psi} \Psi)^{2}+g_{2}\left(\bar{\Psi}_{\gamma_{5}} \Psi\right)^{2}$ were it not for the term $(m+v)$ that replaces the $\sim \operatorname{tr}^{\ominus}\left(\Phi_{K} \otimes \Phi_{K}\right)$ term we would expect. However, the $\Phi_{K}$ in (4.25) are $c$-numbers, while the $\Psi$ in the Lagrangian $\mathscr{L}$ are anticommuting (Grassmann) numbers; it is known [31] that large orders in $g_{R}$ do not follow from such "classical" equations, merely because the "classical" limit of a fermionic field is not the same thing as the classical limit of a bosonic field. We cannot therefore obtain the same "limit exchange" between $1 / N$-governing instantons and $g$-governing instantons, as we had done in the bosonic case.

Our conclusion will be simply that $S_{\text {eff }}$ is probably dominated by the term $1 / g_{R} \int_{0}^{+\infty} w^{2}(r) r d r$, since other evaluable terms behave like $\ln g_{R}$. Large orders behaviour in $1 / N$ is $\mathscr{Z}(K) \sim K ! /\left(-S_{c}\right)^{K}$, where $S_{c}$ is generically complex. The large orders exhibit a phase oscillating behaviour, and therefore the series can be probably resummed by Borel transformation.

\section{Appendix}

We shall here derive the trace identity for $\int_{0}^{+\infty} r \ln m r\left(v^{2}+2 m v+w^{2}\right) d r$, which appears in the effective action after dimensional regularization. We shall here extensively use the relations of differentiation of the potential with respect to the scattering data, which were derived in Sect. III. We recall that we have obtained:

$$
\begin{gathered}
\frac{\delta v(r)}{\delta D(\tau)}=-\frac{1}{r} \frac{\sinh \pi \tau}{2 \pi(D(\tau))^{2}} \operatorname{tr}(\Phi(r, i \tau) \otimes \Phi(r, i \tau)), \\
\frac{\delta v(r)}{\delta J_{K}}=\frac{2}{r} c_{K} \operatorname{tr}\left(\left.\frac{d \Phi}{d J}(r, J)\right|_{J_{K}} \otimes \Phi_{K}\left(r, J_{K}\right)\right), \\
\frac{\delta v(r)}{\delta c_{K}}=\frac{1}{r} \operatorname{tr}\left(\Phi_{K}(r) \otimes \Phi_{K}(r)\right) .
\end{gathered}
$$

The same relations hold for $i w$, except that we have to replace $\operatorname{tr}(a \otimes b)$ $\left(=a_{1} b_{1}+a_{2} b_{2}\right)$ by $\operatorname{tr}^{\ominus}(a \otimes b)\left(=a_{1} b_{1}-a_{2} b_{2}\right)$ in $(1)$. 
We shall also use the linear system:

$$
\left\{\begin{array}{l}
d_{\varrho} \varphi^{(1)}-\frac{J}{\varrho} \varphi^{(1)}=(v+1-i w) \varphi^{(2)} \\
d_{\varrho} \varphi^{(2)}+\frac{J}{\varrho} \varphi^{(2)}=(v+1+i w) \varphi^{(1)}
\end{array} \quad(m \equiv 1),\right.
$$

and the wronskian property:

$$
\frac{d}{d \varrho} W\left(\Phi_{1}(\varrho), \Phi_{2}(\varrho)\right)=\frac{J_{1}-J_{2}}{\varrho}\left(\varphi_{1}^{(1)} \varphi_{2}^{(2)}+\varphi_{1}^{(2)} \varphi_{2}^{(1)}\right) .
$$

Now we shall proceed as follows: Computing the functional derivatives of

$$
Q=\int_{0}^{+\infty} r \ln r\left(v^{2}+2 m v+w^{2}\right) d r
$$

we shall obtain from (1) expressions that can be computed exactly, thanks to (2) and (3), in terms of the scattering data. A trivial integration then leads to the solution.

Let us compute the $c_{K}$ dependence. We get:

$$
\frac{\delta Q}{\delta c_{K}}=\int_{0}^{+\infty} r \ln r\left[(2 v+2) \frac{\delta v}{\delta c_{K}}-2 i w\left(\frac{\delta i w}{\delta c_{K}}\right)\right] d r .
$$

Using (1c), we get:

$$
\frac{\delta Q}{\delta c_{K}}=2 \int_{0}^{+\infty} r \ln r \cdot \frac{1}{r} \cdot\left[(v+1-i w) \varphi_{K}^{(1)} \varphi_{K}^{(1)}+(v+1+i w) \varphi_{K}^{(2)} \varphi_{K}^{(2)}\right] d r .
$$

Using now (2) gives:

$$
\begin{aligned}
\frac{\delta Q}{\delta c_{K}} & =2 \int_{0}^{+\infty} \ln r\left(d \varrho \varphi_{K}^{(1)} \varphi_{K}^{(2)}+d \varrho \varphi_{K}^{(2)} \varphi_{K}^{(1)}\right) d r \\
& =2 \int_{0}^{+\infty} \ln r \frac{d}{d r}\left(\varphi_{K}^{(1)} \varphi_{K}^{(2)}\right) \\
& =2 \int_{0}^{+\infty} \frac{\varphi_{K}^{(1)} \varphi_{K}^{(2)}}{r} d r \quad \text { after integration by parts. }
\end{aligned}
$$

Hence $\frac{\delta Q}{\delta c_{K}}=-1 / c_{K}$. Note that $Q$, as a functional of the potentials, only depends analytically on the S.D. and not on their complex conjugate. Hence

$$
Q=-\sum_{K} \ln c_{K}+\mathscr{F}\left(D(\tau), J_{K}\right)
$$

We have here given in detail the procedure of computing the functional derivative and integrating with respect to the S.D. For the two other derivations, we shall merely give the framework of computation,

$$
\frac{\delta Q}{\delta J_{K}}=\int_{0}^{+\infty} r \ln r\left[(2 v+2) \frac{\delta v}{\delta J_{K}}+(-2 i w) \frac{\delta i w}{\delta J_{K}}\right] d r .
$$


Using (1) and (2), together with the expression of $\Phi$-solutions near the origin

$$
\left\{\begin{array}{l}
\varphi^{(1)} \sim-F^{-}(J) \varrho^{J}+F^{+}(J) \cdot \varrho^{(1-J)} \cdot \alpha^{(1)}, \\
\varphi^{(2)} \sim F^{+}(J) \varrho^{-J}-F^{-}(J) \varrho^{1+J} \cdot \alpha^{(2)}
\end{array}\right.
$$

and the definition $\left.\frac{d \Phi(r, J)}{d J} \equiv \frac{\Phi\left(r, J^{\prime}\right)-\Phi(r, J)}{J^{\prime}-J}\right|_{J \rightarrow J^{\prime}}$, we obtain:

$$
\frac{\delta Q}{\delta J_{K}}=-\frac{F^{\prime \prime}\left(J_{K}\right)}{F^{\prime}\left(J_{K}\right)}
$$

An exactly similar computation for $\frac{\delta Q}{\delta D(\tau)}$, using the same tricks for integrating terms like $\sim \int_{0}^{+\infty} v \cdot \varphi\left(J_{1}\right) \cdot \varphi\left(J_{1}\right) \frac{d r}{r}$, leads to:

$$
\frac{\delta Q}{\delta D(\tau)}=\frac{1}{2 \pi D(\tau)} \cdot\left(\frac{F_{+}^{\prime}(i \tau)}{F_{+}(i \tau)}-\frac{F_{-}^{\prime}(i \tau)}{F_{-}(i \tau)}\right)
$$

Using the dispersion relations obtained in Sect. III for the Jost functions $F^{+}$and $F^{-}$allows one to integrate these two differential equations, not forgetting that the independent variables are $\left\{c_{K}, J_{K}, D(\tau)\right\}$ :

$$
\begin{aligned}
Q= & \int_{0}^{+\infty} r \ln r\left(v^{2}+2 m v+w^{2}\right) d r \\
= & -\frac{1}{4 \pi^{2}} \stackrel{f}{f} \underset{-\infty}{f} \frac{d \tau d \tau^{\prime}}{\left(\tau-\tau^{\prime}\right)^{2}} \ln D(\tau) \ln D\left(\tau^{\prime}\right)+\frac{1}{2 \pi} \\
& \cdot \int_{-\infty}^{+\infty} d \tau\left[2 \ln 2+\Psi\left(\frac{1}{2}+i \tau\right)+\Psi\left(\frac{1}{2}-i \tau\right)-2 \sum_{K} \frac{\operatorname{sgn}\left(\operatorname{Re} J_{K}\right)}{J_{K}-i \tau}\right] \ln D(\tau) \\
& -2 \ln 2 \sum_{K} \operatorname{sgn}\left(\operatorname{Re} J_{K}\right) \cdot J_{K}-\sum_{K} 2 \ln \Gamma\left(\frac{1}{2}+J_{K}\left(\operatorname{sgn} \operatorname{Re} J_{K}\right)\right) \\
& -2 \sum_{K \neq K^{\prime}} \operatorname{sgn}\left(\operatorname{Re} J_{K}\right) \operatorname{sgn}\left(\operatorname{Re} J_{K}^{\prime}\right) \ln \left(J_{K}-J_{K}^{\prime}\right)-\sum_{K} \ln c_{K} .
\end{aligned}
$$

Acknowledgements. The authors wish to thank Natan Andrei for fruitful discussions.

\section{References}

1a. Schwinger, J.: On the Green's functions of quantized fields. I. Proc. Nat. Acad. Sci. USA 37, $452(1951)$

b. Jona-Lasinio, G.: Relativistic field theories with symmetry-breaking solutions. Nuov. Cimento 34, 1790 (1964)

c. Coleman, S., Weinberg, E.: Radiative corrections as the origin of spontaneous symmetry breaking. Phys. Rev. D 7, 1888 (1973)

2. de Vega, H.: Large orders in the $1 / N$ perturbation theory by inverse scattering in one dimension. Commun. Math. Phys. 70, 29 (1979)

3. Avan, J.: $1 / N$ series for quantum anharmonic oscillator eigenvalues and Green functions. Nucl. Phys. B 237, 159 (1984)

4. Avan, J., de Vega, H.: $1 / N$ expansion for invariant potentials in quantum mechanics. Nucl. Phys. B 224, 61 (1983) 
5. Avan, J., de Vega, H.: Classical solutions by inverse scattering transformation in any number of dimensions. I. The gap equation and effective action. Phys. Rev. D 29, 2891 (1984)

6. Avan, J., de Vega, H.: Classical solutions by inverse scattering transformation in any number of dimensions. II. Instantons and large orders of the $1 / N$ series for the $\left(\phi^{2}\right)^{2}$ theory in $v$

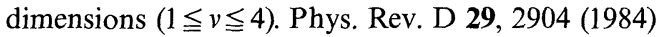

7. de Vega, H.: The inverse scattering transformation in the angular momentum plane. Commun. Math. Phys. 81, 313 (1981)

8. de Vega, H., Schaposnik, F.: Nonuniform external fields and vacuum properties in a twodimensional gauge theory. Phys. Rev. D 26, 2814 (1982)

9. de Vega, H.: The non-linear sigma model in the $1 / N$ expansion and the inverse scattering transformation in the angular momentum. Phys. Lett. B 98, 280 (1981)

10a. Lipatov, L.N.: Divergence of the perturbation-theory series and pseudoparticles. JETP Lett. 25, 104 (1977)

b. Zinn-Justin, J.: Perturbation series at large orders in quantum mechanics and field theories: Application to the problem of resummation. Phys. Rep. 70, 109 (1981)

c. Brézin, E., Le Guillou, J.C., Zinn-Justin, J.: Perturbation theory at large order. I. The $\phi^{2 N}$ interaction. Phys. Rev. D 15, 1544, 1558 (1978)

d. Zinn-Justin, J.: Les Houches lectures. Recent advances in quantum field theory, Stora, R., Zuber, J.B. (eds.). Amsterdam: North-Holland 1983

11. Köberlé, R., Kurak, V., Swieca, A.: Scattering theory and $1 / N$ expansion in the Chiral GrossNeveu model. Phys. Rev. D 20, 897 (1979)

12. Coleman, S., Glaser, V., Martin, A.: Commun. Math. Phys. 58, 211 (1978)

13. Neveu, A., Papanicolaou, N.: Integrability of the classical $\left[\bar{\psi}_{i} \psi_{i}\right]_{2}^{2}$ and $\left[\bar{\psi}_{i} \psi_{i}\right]_{2}^{2}-\left[\bar{\psi}_{i} \gamma_{5} \psi_{i}\right]_{2}^{2}$ interactions. Commun. Math. Phys. 58, 31 (1978)

14. Karowski, M., Thun, H.J.: Complete $S$-matrix of the $O(2 N)$ Gross-Neveu model. Nucl. Phys. B 190, 61 (1981)

15. Karowski, M., Weisz, P.: Exact form factors in $(1+1)$-dimensional field theoretic models with soliton behaviour. Nucl. Phys. B 139, 455 (1978)

16. Abdalla, E., Berg, B., Weisz, P.: More about the $S$-matrix of the chiral $S U(N)$ Thirring model. Nucl. Phys. B 157, 387 (1979)

17. Mitter, P.K., Weisz, P.: Asymptotic scale invariance in a massive Thirring model with $U(n)$ symmetry. Phys. Rev. D 8, 4410 (1973)

18. Gross, D.J., Neveu, A.: Dynamical symmetry breaking in asymptotically free field theories. Phys. Rev. D 10, 3235 (1974)

19a. t'Hooft, G., Veltman, M.: Regularization and renormalization of gauge fields. Nucl. Phys. B 44, 189 (1972)

b. Bollini, C.G., Giambiagi, J.J.: Lowest order "divergent" graphs in $v$-dimensional space. Phys. Lett. B 40, 566 (1972)

c. Bollini, C.G., Giambiagi, J.J.: Dimensional renormalization: The number of dimensions as a regularizing parameter. Nuovo Cimento B 12, 20 (1972)

20. Boerner, H.: Representation of groups, pp. 269-273. Amsterdam: North-Holland 1963

21. Stratonovitch, R.L.: Dokl. Akad. Nauk. SSSR 115, 1097 (1957)

22. Berg, B., Weisz, P.: Exact $S$-matrix of the chiral invariant $S U(N)$ Thirring model. Nucl. Phys. B 146, 205 (1978)

23. Coleman, S.: There are no Goldstone bosons in two dimensions. Commun. Math. Phys. 31, 259 (1973)

24. Roskies, R., Schaposnik, F.: Comment on Fujikawa's analysis applied to the Schwinger model. Phys. Rev. D 23, 558 (1981)

25. Magnus, W., Oberhettinger, R., Soni, R.: Formulas and theorems for the special functions of mathematical physics. Berlin, Heidelberg, New York: Springer 1966

26a. Spencer, T.: The Lipatov argument. Commun. Math. Phys. 74, 273 (1980)

b. Breen, S.: Leading large order asymptotics for $\left(\phi^{4}\right)_{2}$ perturbation theory. Commun. Math. Phys. 92, 179 (1981)

c. Magnen, J., Rivasseau, V.: Preprint CPhT 652-0285 
27. Ablowitz, M., Kaup, D., Newell, A., Segur, H.: The inverse scattering transform - Fourier analysis for nonlinear problems. Stud. Appl. Math., vol. III 4, 249 (1974)

28a. Birman, M.S., Krein, M.O.: Sov. Phys. Dokl. 3, 740 (1962)

b. Buslaev, V.S.: Topics in Math. Phys., Vol. I, p.69, Birman, M.Sh. (ed.). New York: Consultants Bureau 1968

29. Gradhshteyn, I.S., Ryzhik, I.M.: Table of integrals, series, and products. New York: Academic Press 1980, 8/362

30. Zamolodchikov, A.B., Zamolodchikov, Al.B.: Ann. Phys. (NY) 80, 253 (1979)

31. The problems of large orders in the coupling constant for fermionic theories are discussed for instance in:

a. Parisi, G.: Asymptotic estimates in perturbation theory with fermions. Phys. Lett. B 66, 382 (1977)

b. Fainberg, V., Pakshaev, I.: On the fermion contribution to instantons. Phys. Lett. B 77, 208 (1978)

c. Bogomolnyi, V., Kubishin, Y.: Asymptotic estimates for graphs with a fixed number of fermion loops in quantum electrodynamics. The choice of the form of the steepest descent solutions. Sov. J. Nucl. Phys. 34, 853 (1981) and: Asymptotic estimates for diagrams with a fixed number of fermion loops in quantum electrodynamics. The extremal configurations with the symmetry group $O(2) \otimes O(3)$. 35, $114(1982)$

d. Itzykson, C., Parisi, G., Zuber, J.B.: Asymptotic estimates in quantum electrodynamics. Phys. Rev. D 16, 996 (1978)

32. Feldman, J., Magnen, J., Rivasseau, V., Sénéor, R.: Preprint CPhT 644-0185

We wish to thank V. Rivasseau for indicating to us refs. [26c] and [32].

Communicated by G. Mack

Received March 14, 1985 